\title{
A variety of dynamic inequalities on time scales with retar- dation
}

\author{
A. A. El-Deeb ${ }^{a}$, Wing-Sum Cheung, ${ }^{b, *}$ \\ ${ }^{a}$ Department of Mathematics, Faculty of Science, Al-Azhar University, Nasr City (11884), Cairo, Egypt. \\ ${ }^{b}$ Department of Mathematics, The University of Hong Kong, Pokfulam Road, Hong Kong. \\ Communicated S. H. Wu
}

\begin{abstract}
In this paper, we will prove some new nonlinear retarded dynamic inequalities of Gronwall-Bellman type on time scales. These inequalities are of new forms compared with the existing results so far in the literature, which can be used as effective tools in the study of certain nonlinear retarded dynamic equations. Some special cases of our results contain continuous Gronwall-type inequalities and their discrete analogues. We also indicate some application examples to illustrate our results at the end.
\end{abstract}

Keywords: Gronwall's inequality, Young's inequality, time scales.

2010 MSC: 26A15, 39A12, 34A12, 34A40, 26D10, 26D15, 26D20.

(C)2018 All rights reserved.

\section{Introduction}

The Gronwall inequality [12] states that if $f$ and $v$ are real-valued nonnegative continuous functions defined on $\mathbb{R}_{+}=[0, \infty)$ with a positive constant $v_{0}$, then

$$
v(t) \leqslant v_{0}+\int_{0}^{t} f(s) v(s) d s, \forall t \in \mathbb{R}_{+},
$$

implies

$$
v(t) \leqslant v_{0} \exp \left(\int_{0}^{t} f(s) d s\right), \forall t \in \mathbb{R}_{+} .
$$

As a generalization of (1.1), Bellman [7] proved that: If $v, f, a, \in C\left(\mathbb{R}_{+}, \mathbb{R}_{+}\right)$and $a$ is nondecreasing, then the inequality

$$
v(t) \leqslant a(t)+\int_{0}^{t} f(s) v(s) d s, \forall t \in \mathbb{R}_{+}
$$

\footnotetext{
${ }^{*}$ Corresponding author

Email addresses: ahmedeldeeb@azhar.edu.eg (A. A. El-Deeb), wscheung@hku.hk (Wing-Sum Cheung)

doi: $10.22436 /$ jnsa.011.10.07
}

Received: 2017-12-07 Revised: 2017-12-21 Accepted: 2018-03-25 
implies

$$
v(t) \leqslant a(t) \exp \left(\int_{0}^{t} f(s) d s\right), \forall t \in \mathbb{R}_{+} .
$$

In [26], Pachpatte established the discrete version of (1.2) and in particular he proved that if $u(n), a(n)$, $b(n)$ are nonnegative sequences defined for $n \in \mathbb{N}_{0}$ and $a(n)$ is nondecreasing for $n \in \mathbb{N}_{0}$, and if

$$
u(n) \leqslant a(n)+\sum_{s=0}^{n-1} f(n) u(n), n \in \mathbb{N}_{0}
$$

then

$$
u(n) \leqslant a(n) \prod_{s=0}^{n-1}[1+f(n)], n \in \mathbb{N}_{0} .
$$

Since the discovery of these inequalities, many inequalities which deal with new proofs, various generalizations and extensions have presented in the literature. Such inequalities are important handy tools to obtain various estimates in the theory of differential and difference equations; see for example [1$4,8,11,20,22-24]$.

In [10], Bohner and Peterson introduced a dynamic inequality on a time scale $\mathbb{T}$ which unifies the continuous version inequality (1.2) and the discrete version inequality (1.3) as follows: If $\chi, \delta$ are right dense continuous functions and $\gamma \geqslant 0$ is regressive and right dense continuous function, then

$$
\chi(t) \leqslant \delta(t)+\int_{t_{0}}^{t} \chi(\eta) \gamma(s) \Delta \eta, \forall t \in \mathbb{T}
$$

implies

$$
\chi(t) \leqslant \delta(t)+\int_{t_{0}}^{t} e_{\gamma}(t, \sigma(\eta)) \delta(\eta) \gamma(s) \Delta \eta, \forall t \in \mathbb{T} .
$$

Pachpatte [25] established explicit bounds to the solution of the following integral inequality:

$$
u^{p}(t) \leqslant a(t)+b(t) \int_{0}^{t}\left[g(s) u^{p}(s)+h(s) u(s)\right] d s, t \in \mathbb{R}_{+},
$$

where $u, a, b, g, h$ are real-valued nonnegative continuous functions defined on $\mathbb{R}_{+}$and $p>1$ is a real constant.

Also in the same paper [25], for a real-valued positive continuous and nondecreasing function c defined on $\mathbb{R}_{+}$, Pachpatte studied the following inequalities:

$$
\begin{aligned}
& u^{p}(t) \leqslant c^{p}(t)+b(t) \int_{0}^{t}\left[g(s) u^{p}(s)+h(s) u(s)\right] d s, t \in \mathbb{R}_{+}, \\
& u^{p}(t) \leqslant a(t)+b(t) \int_{0}^{t} k(t, s)\left[g(s) u^{p}(s)+h(s) u(s)\right] d s, t \in \mathbb{R}_{+},
\end{aligned}
$$

and

$$
u^{p}(t) \leqslant a(t)+b(t) \int_{0}^{t} f(s, u(s)) d s, t \in \mathbb{R}_{+},
$$

where $k(t, s)$ and its derivative $\frac{\partial}{\partial t} k(t, s)$ are real-valued nonnegative continuous functions for $0 \leqslant s \leqslant t \leqslant$ $\infty$, and $f: \mathbb{R}_{+}^{2} \rightarrow \mathbb{R}_{+}$is a continuous function.

On the other hand, Pachpatte [25] also investigated the following discrete analogues of (1.4), (1.5), (1.6), and (1.7).

$$
u^{p}(n) \leqslant a(n)+b(n) \sum_{s=n_{0}}^{n-1}\left[g(n) u^{p}(n)+h(n) u(n)\right], n \in \mathbb{N}_{0}
$$




$$
\begin{aligned}
& u^{p}(n) \leqslant c^{p}(n)+b(n) \sum_{s=n_{0}}^{n-1}\left[g(n) u^{p}(n)+h(n) u(n)\right], n \in \mathbb{N}_{0}, \\
& u^{p}(n) \leqslant a(n)+b(n) \sum_{s=n_{0}}^{n-1} k(n, s)\left[g(n) u^{p}(n)+h(n) u(n)\right], n \in \mathbb{N}_{0}, \\
& u^{p}(n) \leqslant a(n)+b(n) \sum_{s=n_{0}}^{n-1} F(s, u(s)), n \in \mathbb{N}_{0},
\end{aligned}
$$

where $u(n), a(n), b(n), g(n), h(n)$, and $c(n)$ are real-valued nonnegative sequences, and $F: \mathbb{N}_{0} \times \mathbb{R}_{+} \rightarrow$ $\mathbb{R}_{+}$, and $k(n, s), \Delta_{1} k(n, s)$ are real-valued nonnegative functions for $n \leqslant s \leqslant n, n \in \mathbb{N}_{0}$.

In [16], Li studied the following nonlinear delay integral inequalities of (1.4) and (1.7):

$$
u^{p}(t) \leqslant a(t)+b(t) \int_{0}^{t}\left[f(s) u^{p}(s-\tau)+g(s) u(s)+h(s)\right] d s, t \in \mathbb{R}_{+},
$$

and

$$
u^{p}(t) \leqslant a(t)+b(t) \int_{0}^{t} L(s, u(s-\tau)) d s, t \in \mathbb{R}_{+},
$$

with the initial condition:

$$
\begin{cases}u(t)=\phi(t), & t \in[-\tau, 0], \\ \phi(t-\tau) \leqslant(a(t))^{\frac{1}{p}}, & \forall t \in \mathbb{R}_{+}, \quad t-\tau \leqslant 0,\end{cases}
$$

where $p>1$ and $\tau \in \mathbb{R}_{+}$are constants, $\phi(t) \in \mathrm{C}\left([-\tau, 0], \mathbb{R}_{+}\right)$, and $\mathrm{L} \in \mathrm{C}\left(\mathbb{R}_{+}^{2}, \mathbb{R}_{+}\right)$.

On the other hand, in the same paper [16], the author also investigated the following discrete analogues of (1.8) and (1.9):

$$
u^{p}(n) \leqslant a(n)+b(n) \sum_{s=0}^{n-1}\left[f(s) u^{p}(s-\sigma)+g(s) u(s)+h(s)\right], n \in \mathbb{N}_{0}
$$

and

$$
u^{p}(n) \leqslant a(n)+b(n) \sum_{s=0}^{n-1} v(s, u(s-\sigma)), n \in \mathbb{N}_{0},
$$

with the initial condition:

$$
\left\{\begin{array}{lc}
\mathrm{u}(\mathrm{n})=\psi(\mathrm{n}), & \mathrm{n} \in\{-\sigma, \ldots,-1,0\}, \\
\psi(\mathrm{n}-\sigma) \leqslant(\mathrm{a}(\mathrm{n}))^{\frac{1}{p}}, & \forall \mathrm{n} \in \mathbb{N}_{0}, \quad \mathrm{n}-\sigma \leqslant 0,
\end{array}\right.
$$

where $p>1$ and $\sigma \in \mathbb{N}_{0}$ are constants, $\psi(n) \in \mathbb{R}_{+}$, and $V: \mathbb{N}_{0} \times \mathbb{R}_{+} \rightarrow \mathbb{R}_{+}$.

In recent years, the study of dynamic inequalities of one variable of Gronwall-type on time scales has received a lot of attention, we refer the reader to the papers $[5,6,10,14,15,17-19,27-29]$ and the references cited therein.

The general idea is to prove a result for a dynamic inequality where the domain of the unknown function is a time scale $\mathbb{T}$, which may be an arbitrary closed subset of the real numbers $\mathbb{R}$. The cases when the time scale is equal to the reals or to the integers represent the classical theories of integral and of discrete inequalities. The calculus on time scales has been introduced by Hilger [13] in order to unify discrete and continuous analysis. The three most popular examples of calculus on time scales are differential calculus, difference calculus, and quantum calculus, when $\mathbb{T}=\mathbb{R}, \mathbb{T}=\mathbb{N}$, and $\mathbb{T}=q^{N_{0}}=\left\{q^{t}\right.$ : $\left.t \in N_{0}\right\}$, where $q>1$. For the general basic ideas and background, we refer to $[9,10]$ which summarize and organize much of the time scale calculus. 
The main objective of this paper is to prove new delta dynamic inequalities on an arbitrary time scale $\mathbb{T}$ with several retardation that resemble both the discrete and the continuous inequalities presented in $[16,25]$. We also provide a more useful and explicit bound than in [16, 25]. The paper is organized in the following way. In Section 2, we give some basic concepts of the calculus on time scales. In Section 3 , we state and prove the main results, which can be used as effective tools in the study of certain nonlinear retarded dynamic equations. In Section 4, we include some applications to illustrate the usefulness of our results.

\section{Preliminaries and lemmas on time scales}

In this section, we will give some preliminaries on calculus of time scales and the basic lemmas that we will apply to obtain the main results in this paper. We assume throughout that $\mathbb{T}$ has the topology inherited from the standard topology on the real numbers $\mathbb{R}$. For $t \in \mathbb{T}$, first we define the forward jump operator $\sigma: \mathbb{T} \rightarrow \mathbb{T}$ by:

$$
\sigma(t):=\inf \{s \in \mathbb{T}: s>t\},
$$

and second, the backward jump operator $\rho: \mathbb{T}: \rightarrow \mathbb{T}$ by:

$$
\rho(\mathrm{t}):=\sup \{s \in \mathbb{T}: \mathrm{s}<\mathrm{t}\}
$$

In this definition, we put $\inf \emptyset=\sup \mathbb{T}$, where $\emptyset$ is the empty set (i.e., $\sigma(t)=t$ if $\mathbb{T}$ has a maximum $m$ ) and $\sup \emptyset=\inf \mathbb{T}$ (i.e., $\mathbb{T}$ has a maximum $l$ ). A point $t \in \mathbb{T}$ with $\inf \mathbb{T}<t<\sup \mathbb{T}$ is said to be left-dense if $\rho(t)=t$ and is right-dense if $\sigma(t)=t$, points that are simultaneously right-dense and left-dense are said to be dense, is left-scattered if $\rho(t)<t$ and right-scattered if $\sigma(t)>t$, points that are simultaneously right-scattered and left-scattered are said to be isolated. A function $g: \mathbb{T} \rightarrow \mathbb{R}$ is said to be right-dense continuous (rd-continuous) provided $g$ is continuous at right-dense points and at left-dense points in $\mathbb{T}$, left hand limits exist and are finite. The set of all such rd-continuous functions is denoted by $\mathrm{C}_{\mathrm{rd}}(\mathbb{T})$. A function $\mathrm{f}: \mathbb{T} \rightarrow \mathbb{R}$ is said to be left-dense continuous (ld-continuous) provided $\mathrm{f}$ is continuous at left-dense points and at right-dense points in $\mathbb{T}$, right-hand limits exist and are finite. The set of all such ld-continuous functions is denoted by $\mathrm{C}_{\mathrm{ld}}(\mathbb{T})$.

The forward and backward graininess functions $\mu$ and $\nu$ for a time scale $\mathbb{T}$ are defined by $\mu(t):=$ $\sigma(t)-t$ and $v(t)=t-\rho(t)$, respectively.

Given a time scale $\mathbb{T}$, we introduce the sets $\mathbb{T}^{k}, \mathbb{T}_{k}$, and $\mathbb{T}_{k}^{k}$ as follows. If $\mathbb{T}$ has a left-scattered maximum $t_{1}$, then $\mathbb{T}^{k}=\mathbb{T}-\left\{t_{1}\right\}$, otherwise $\mathbb{T}^{k}=\mathbb{T}$. If $\mathbb{T}$ has a right-scattered minimum $t_{2}$, then $\mathbb{T}^{\mathrm{k}}=\mathbb{T}-\left\{\mathrm{t}_{2}\right\}$, otherwise $\mathbb{T}^{\mathrm{k}}=\mathbb{T}$. Finally $\mathbb{T}_{\mathrm{k}}^{\mathrm{k}}=\mathbb{T}^{\mathrm{k}} \cap \mathbb{T}_{\mathrm{K}}$.

Let $f: \mathbb{T} \rightarrow \mathbb{R}$ be a real valued function on a time scale $\mathbb{T}$, then for all $t \in \mathbb{T} \kappa$, we define $f^{\Delta}(t)$ to be the number (if it exists) with the property that given any $\varepsilon>0$ there is a neighborhood $U$ of $t$ such that $\forall \mathrm{s} \in \mathrm{U}$,

$$
\left|[f(\sigma(t))-f(s)]-f^{\Delta}(t)[\sigma(t)-s]\right| \leqslant \varepsilon|\sigma(t)-s|, \quad \forall s \in U .
$$

For $f: \mathbb{T} \rightarrow \mathbb{R}$, we define the function $f^{\sigma}: \mathbb{T} \rightarrow \mathbb{R}$ by $f^{\sigma}(t)=f(\sigma)$ for all $t \in \mathbb{T}$, that is $f^{\sigma}(t)=f \circ \sigma$. Similarly, we define the function $f^{\rho}: \mathbb{T} \rightarrow \mathbb{R}$ by $f^{\rho}(t)=f(\sigma)$, for all $t \in \mathbb{T}$, that is $f^{\rho}(t)=f \circ \sigma$. A time scale $\mathbb{T}$ is said to be regular if the following two conditions are satisfied simultaneously: $(1) \sigma(\rho(t))=t$, and (2) $\rho(\sigma(t))=t, \forall t \in \mathbb{T}$. The product and quotient rules for the derivative of the product $f g$ and the quotient $\mathrm{f} / \mathrm{g}$ (where $\mathrm{gg}^{\sigma} \neq 0$, here $\mathrm{g}^{\sigma}=\mathrm{g} \circ \sigma$ ) of two differentiable function $\mathrm{f}$ and $\mathrm{g}$, are given as the following:

$$
(f g)^{\Delta}(t)=f^{\Delta}(t) g(t)+f(\sigma(t)) g^{\Delta}(t)=f(t) g^{\Delta}(t)+f^{\Delta}(t) g(\sigma(t)),
$$

and

$$
\left(\frac{f}{g}\right)^{\Delta}(t)=\frac{f^{\Delta}(t) g(t)-f(t) g^{\Delta}(t)}{g(t) g(\sigma(t))}
$$


A function $F: \mathbb{T} \rightarrow \mathbb{R}$, is called a delta antiderivative of $f: \mathbb{T} \rightarrow \mathbb{R}$ provided that $F^{\Delta}=f(t)$ holds for all $t \in \mathbb{T}^{k}$, and the delta integral of $f$ is defined by

$$
\int_{a}^{b} f(t) \Delta t=F(b)-F(a) .
$$

We will frequently use the following useful relations between calculus on time scales $\mathbb{T}$ and differential calculus on $\mathbb{R}$, difference calculus on $\mathbb{Z}$, and quantum calculus on $\mathrm{q}_{0}^{\mathbb{N}}$ :

(i) if $\mathbb{T}=\mathbb{R}$, then

$$
\sigma(t)=t, \mu(t)=0, f^{\Delta} t=f^{\prime}(t), \int_{a}^{b} f(t) \Delta t=\int_{a}^{b} f(t) d t
$$

(ii) if $\mathbb{T}=\mathbb{Z}$, then

$$
\sigma(t)=t+1, \mu(t)=1, f^{\Delta} t=\Delta f(t), \int_{a}^{b} f(t) \Delta t=\sum_{t=a}^{b-1} f(t)
$$

(iii) and if $\mathbb{T}=\mathrm{q}^{\mathbb{N}_{0}}=\left\{\mathrm{t}: \mathrm{t}=\mathrm{q}^{\mathrm{k}}, \mathrm{k} \in \mathbb{N}_{0}, \mathrm{q}>1\right\}$, then

$$
\sigma(t)=q t, \mu(t)=(q-1) t, \int_{a}^{b} f(t) \Delta t=(q-1) \sum_{k=\log _{q}(a)}^{\log _{q}(b)-1} q^{k} f\left(q^{k}\right), \forall a, b \in q^{\mathbb{N}_{0}}
$$

It can be shown (see [10]) that if $g \in C_{r d}(T)$, then the Cauchy integral $G(t):=\int_{t_{0}}^{t} g(s) \Delta s$ exists, $t_{0} \in \mathbb{T}$, and satisfies $G^{\Delta}(t)=g(t), t \in \mathbb{T}$. An infinite integral is defined as

$$
\int_{a}^{\infty} f(t) \Delta(t)=\lim _{b \rightarrow \infty} \int_{a}^{b} f(t) \Delta t
$$

Now, we will give the definition of the generalized exponential functions and its derivatives. We say that $p: \mathbb{T}^{k} \rightarrow \mathbb{R}$ is regressive provided $1+\mu(t) p(t) \neq 0$ for all $t \in \mathbb{T}^{k}$. We define $\Re$ as the set of all regressive and rd-continuous functions, and define the set $\mathfrak{R}^{+}$the set of all positive elements of $\mathfrak{R}$, that is $\mathfrak{R}^{+}=\{p \in \mathfrak{R}: 1+\mu(t) p(t)>0, \forall t \in \mathbb{T}\}$. The set of all regressive functions on a time scale $\mathbb{T}$ forms an Abelian group under the addition $\oplus$ defined by $p \oplus q=p+q+\mu p q$. If $p \in \mathfrak{R}^{+}$, then we can define the exponential function by

$$
e_{p}(t, s)=\exp \left(\int_{s}^{t} \xi_{\mu(\tau)}(p(\tau)) \Delta \tau\right), \forall t \in \mathbb{T}, s \in \mathbb{T}^{k},
$$

where $\xi_{h}(z)$ is the cylinder transformation, which is defined by

$$
\xi_{h}(z)= \begin{cases}\frac{\log (1+h z)}{h}, & h \neq 0, \\ z, & h=0 .\end{cases}
$$

If $p \in \Re$, then $e_{p}(t, s)$ is real-valued and nonzero on $\mathbb{T}$. If $p \in \mathfrak{R}^{+}$, then $e_{\mathfrak{p}}\left(t, t_{0}\right)$ is always positive.

Note that

- if $\mathbb{T}=\mathbb{R}$, then,

$$
e_{a}\left(t, t_{0}\right)=\exp \left(\int_{t_{0}}^{t} a(s) d s\right)
$$

- if $\mathbb{T}=\mathbb{Z}$, then,

$$
e_{\mathrm{a}}\left(\mathrm{t}, \mathrm{t}_{0}\right)=\prod_{s=\mathrm{t}_{0}}^{\mathrm{t}-1}(1+\mathrm{a}(\mathrm{s}))
$$


- if $\mathbb{T}=q^{\mathbb{N}_{0}}$, then,

$$
e_{a}\left(t, t_{0}\right)=\prod_{s=t_{0}}^{t-1}(1+(q-1) s a(s)) .
$$

In the following we present the basic lemmas that will be needed in the proof of our main results.

Lemma 2.1 ([10]). If $p, q \in \Re$ and $t_{0} \in \mathbb{T}$, then

1. $e_{p}(t, t)=1=e_{0}(t, s)$;

2. $e_{p}(\sigma(t), s)=(1+\mu(t) p(t)) e_{p}(t, s)$;

3. if $\mathrm{p} \in \mathfrak{R}^{+}$, then $e_{\mathrm{p}}\left(\mathrm{t}, \mathrm{t}_{0}\right)>0, \quad \forall \mathrm{t} \in \mathbb{T}$;

4. $\int_{a}^{b} p(t) e_{p}(c, \sigma(t)) \Delta t=-\int_{a}^{b}\left(e_{p}^{\Delta}(c, \cdot) \Delta\right)=e_{p}(c, a)-e_{p}(c, b)$.

Lemma 2.2 ([10]). If $p \in \Re$, and fix $t \in \mathbb{T}$, then the exponential function $e_{p}\left(t, t_{0}\right)$ is the unique solution of the following initial value problem:

$$
\left\{\begin{array}{l}
y^{\Delta}(t)=p(t) y(t) \\
y\left(t_{0}\right)=1
\end{array}\right.
$$

Lemma 2.3 ([10]). Let $\mathrm{t}_{0} \in \mathbb{T}^{\mathrm{k}}$ and $\mathrm{k}: \mathbb{T} \times \mathbb{T}^{\mathrm{k}} \rightarrow \mathbb{R}$ be continuous at $(\mathrm{t}, \mathrm{t})$, where $\mathrm{t}>\mathrm{t}_{0}, \mathrm{t} \in \mathbb{T}^{\mathrm{k}}$. Assume that $\mathrm{k}^{\Delta}(\mathrm{t},$.$) is r d$-continuous on $\left[\mathrm{t}_{0}, \sigma(\mathrm{t})\right]$. Suppose for any $\varepsilon>0$, there exists a neighborhood $\mathrm{U}$ of $\mathrm{t}$, independent of $\tau \in\left[\mathrm{t}_{0}, \sigma(\mathrm{t})\right]$, such that

$$
\left|[k(\sigma(t), \tau)-k(s, \tau)]-k^{\Delta}(t, \tau)[\sigma(t)-s]\right| \leqslant \varepsilon|\sigma(t)-s|, \quad \forall s \in U .
$$

If $\mathrm{k}^{\Delta}$ denotes the derivative of $\mathrm{k}$ with respect to the first variable, then

$$
f(t)=\int_{t_{0}}^{t} k(t, \tau) \Delta \tau
$$

implies

$$
f^{\Delta}(t)=\int_{t_{0}}^{t} k^{\Delta}(t, \tau) \Delta \tau+k(\sigma(t), t)
$$

Lemma 2.4 ([10]). Suppose $\mathrm{u}, \mathrm{b} \in \mathrm{C}_{\mathrm{rd}}, \mathrm{a} \in \mathfrak{R}^{+}$, then

$$
u^{\Delta}(t) \leqslant a(t) u(t)+b(t), t \geqslant t_{0}, t \in \mathbb{T}^{k},
$$

implies

$$
u(t) \leqslant u\left(t_{0}\right) e_{a}\left(t, t_{0}\right)+\int_{t_{0}}^{t} e_{a}(t, \sigma(\tau)) b(\tau) \Delta \tau, t \geqslant t_{0}, t \in \mathbb{T}^{k}
$$

Lemma 2.5 ([10, p.28, Theorem 1.76]). If $\mathrm{f}^{\Delta}(\mathrm{t}) \geqslant 0$, then $\mathrm{f}(\mathrm{t})$ is nondecreasing.

Lemma 2.6 ([10, p.5, Theorem 1.16]). Assume that $\mathrm{f}: \mathbb{T} \rightarrow \mathbb{R}$ is delta differentiable at $\mathrm{t} \in \mathbb{T}^{\mathrm{k}}$. Then

$$
f^{\sigma}(x)=f(x)+\mu(x) f^{\Delta}(x) .
$$

Lemma 2.7 ([21, Young's inequality]). If $x \geqslant 0, y \geqslant 0$ and $\frac{1}{p}+\frac{1}{q}=1$ with $p>1$, then

$$
x^{\frac{1}{p}} y^{\frac{1}{q}} \leqslant \frac{x}{p}+\frac{y}{q}
$$

Now, we are ready to state and prove the main results in this paper. 


\section{Main results}

In this section, we will prove the main results. For convenience of notation, in the rest of this paper we always assume that $\mathbb{T}_{0}=\left[t_{0}, \infty\right) \cap \mathbb{T}$, where $t_{0} \in \mathbb{T}$, and always assume $\mathbb{T}_{0} \subset \mathbb{T}^{k}$, and $\mathbb{Z}$ denotes the set of integers.

Theorem 3.1. Let $u, a, g, h, b, \xi, f \in C_{r d}\left(\mathbb{T}_{0}, \mathbb{R}^{+}\right), k(t, s), k^{\Delta}(t, s) \in C_{r d}\left(\mathbb{T}_{0} \times \mathbb{T}_{0}, \mathbb{R}^{+}\right)$, and $p \neq 0, p>q>0$, $\mathrm{p}>\mathrm{r}>0, \mathrm{p}>\mathrm{m}>0, \mathrm{p}, \mathrm{q}, \mathrm{r}, \mathrm{m}$ be constants. If

$$
\begin{aligned}
u^{p}(t) \leqslant & a(t)+b(t) \int_{t_{0}}^{t}\left[g\left(\varphi_{1}(s)\right) u^{q}\left(\varphi_{1}(s)\right)+h\left(\varphi_{2}(s)\right) u^{r}\left(\varphi_{2}(s)\right)+k(t, s)\right. \\
& \left.+\int_{0}^{s}\left[f\left(\varphi_{3}(\tau)\right) u^{m}\left(\varphi_{3}(\tau)\right)+\xi(\tau)\right] \Delta \tau\right] \Delta s
\end{aligned}
$$

with the initial condition

$$
\begin{cases}u(t)=\phi(t), & \text { if } t \in\left[\alpha, t_{0}\right] \cap \mathbb{T}, \\ \phi\left(\varphi_{i}(t)\right) \leqslant(a(t))^{1 / p}, & \text { if } \varphi_{i}(t) \leqslant t_{0}, \forall t \in \mathbb{T}_{0}, i=1,2,3,\end{cases}
$$

where $\varphi_{i}(t) \in\left(\mathbb{T}_{0}, \mathbb{T}\right), \varphi_{i}(t)<t,-\infty<\alpha=\inf \left\{\varphi_{i}(t), t \in \mathbb{T}_{0}\right\} \leqslant t_{0}, \phi \in C_{r d}\left(\left[\alpha, t_{0}\right] \cap \mathbb{T}, \mathbb{R}^{+}\right)$, then

$$
u(t) \leqslant\left\{a(t)+b(t) \int_{t_{0}}^{t} \zeta_{1}(s) e_{\eta_{1}}(t, \sigma(s)) \Delta s\right\}^{1 / p}
$$

for all $\mathrm{t} \in \mathbb{T}_{0}$, where

$$
\eta_{1}(t)=b(t)\left(\frac{q}{p} g\left(\varphi_{1}(t)\right)+\frac{r}{p} h\left(\varphi_{2}(t)\right)\right)+\frac{m}{p} \int_{t_{0}}^{t} f\left(\varphi_{3}(\tau)\right) b(t, \tau) \Delta \tau
$$

and

$$
\begin{aligned}
\zeta_{1}(t)= & g\left(\varphi_{1}(t)\right)\left(\frac{p-q}{p}+\frac{q}{p} a(t)\right)+h\left(\varphi_{2}(t)\right)\left(\frac{p-r}{p}+\frac{r}{p} a(t)\right) \\
& +\int_{t_{0}}^{t} f\left(\varphi_{3}(\tau)\right)\left(\frac{p-m}{p}+\frac{m}{p} a(\tau)\right) \Delta \tau+\int_{t_{0}}^{t} \xi(\tau) \Delta \tau+k(\sigma(t), t)+\int_{t_{0}}^{t} k^{\Delta}(t, \tau) \Delta \tau
\end{aligned}
$$

for all $\mathrm{t} \in \mathbb{T}_{0}$.

Proof. Fixing an arbitrary number $\mathrm{T}^{*} \in \mathbb{T}_{0}$, we define a function $\omega(\mathrm{t})$ by:

$$
\begin{aligned}
\omega(t)= & {\left[a\left(T^{*}\right)+b(t) \int_{t_{0}}^{t}\left[g\left(\varphi_{1}(s)\right) u^{q}\left(\varphi_{1}(s)\right)+h\left(\varphi_{2}(s)\right) u^{r}\left(\varphi_{2}(s)\right)+k(t, s)\right.\right.} \\
& \left.\left.+\int_{0}^{s}\left[f\left(\varphi_{3}(\tau)\right) u^{m}\left(\varphi_{3}(\tau)\right)+\xi(\tau)\right] \Delta \tau\right] \Delta s\right]^{1 / p},
\end{aligned}
$$

clearly, $\omega(t)$ is a nonnegative and nondecreasing function, and we have

$$
u(t) \leqslant w(t), \quad t \in\left[t_{0}, T^{*}\right] \cap \mathbb{T} .
$$

If $\varphi_{i}(t) \geqslant t_{0}$ for $t \in\left[t_{0}, T^{*}\right] \cap \mathbb{T}$, since $\varphi_{i}(t) \leqslant t$, we have $\varphi_{i}(t) \in\left[t, T^{*}\right] \cap \mathbb{T}$, and from (3.6), we have

$$
u\left(\varphi_{i}(t)\right) \leqslant \omega\left(\varphi_{i}(t)\right) \leqslant \omega(t), i=1,2,3 .
$$

If $\varphi_{i}(t) \leqslant t_{0}$, from (3.1), we get

$$
u\left(\varphi_{i}(t)\right) \leqslant \varphi\left(\varphi_{i}(t)\right) \leqslant \omega(t)(t), i=1,2,3 .
$$


So from (3.6) and (3.7), we always have

$$
u\left(\varphi_{i}(t)\right) \leqslant \omega(t), i=1,2,3, \forall t \in\left[t_{0}, T^{*}\right] \cap \mathbb{T} .
$$

It follows from (3.5), (3.6), and (3.8) that

$$
\begin{aligned}
\omega^{\mathrm{p}}(\mathrm{t}) \leqslant & a\left(T^{*}\right)+b(t) \int_{\mathrm{t}_{0}}^{\mathrm{t}}\left[g\left(\varphi_{1}(s)\right) \omega^{\mathrm{q}}\left(\varphi_{1}(\mathrm{~s})\right)+\mathrm{h}\left(\varphi_{2}(\mathrm{~s})\right) \omega^{\mathrm{r}}\left(\varphi_{2}(\mathrm{~s})\right)+\mathrm{k}(\mathrm{t}, \mathrm{s})\right. \\
& \left.+\int_{0}^{s}\left[\mathrm{f}\left(\varphi_{3}(\tau)\right) \omega^{\mathrm{m}}\left(\varphi_{3}(\tau)\right)+\xi(\tau)\right] \Delta \tau\right] \Delta s \\
\leqslant & a\left(T^{*}\right)+b(\mathrm{t}) \int_{\mathrm{t}_{0}}^{\mathrm{t}}\left[g\left(\varphi_{1}(\mathrm{~s})\right) \omega^{\mathrm{q}}(\mathrm{s})+\mathrm{h}\left(\varphi_{2}(\mathrm{~s})\right) \omega^{\mathrm{r}}(\mathrm{s})+\mathrm{k}(\mathrm{t}, \mathrm{s})\right. \\
& \left.+\int_{0}^{s}\left[\mathrm{f}\left(\varphi_{3}(\tau)\right) \omega^{\mathrm{m}}(\tau)+\xi(\tau)\right] \Delta \tau\right] \Delta s
\end{aligned}
$$

for all $t \in\left[t_{0}, T^{*}\right] \cap \mathbb{T}$. By taking $t=T^{*}$, we get

$$
\begin{aligned}
\omega^{p}\left(T^{*}\right) \leqslant & a\left(T^{*}\right)+b\left(T^{*}\right) \int_{t_{0}}^{T^{*}}\left[g\left(\varphi_{1}(s)\right) \omega^{q}(s)+h\left(\varphi_{2}(s)\right) \omega^{r}(s)+k\left(T^{*}, s\right)\right. \\
& \left.+\int_{0}^{s}\left[f\left(\varphi_{3}(\tau)\right) \omega^{m}(\tau)+\xi(\tau)\right] \Delta \tau\right] \Delta s
\end{aligned}
$$

for all $t \in\left[t_{0}, T^{*}\right] \cap \mathbb{T}$. But we know that $T^{*} \in \mathbb{T}_{0}$ is arbitrary, from (3.9), we have

$$
\begin{aligned}
\omega^{p}(t) \leqslant & a(t)+b(t) \int_{t_{0}}^{t}\left[g\left(\varphi_{1}(s)\right) \omega^{q}(s)+h\left(\varphi_{2}(s)\right) \omega^{r}(s)+k(t, s)\right. \\
& \left.+\int_{t_{0}}^{s}\left[f\left(\varphi_{3}(\tau)\right) \omega^{m}(\tau)+\xi(\tau)\right] \Delta \tau\right] \Delta s
\end{aligned}
$$

for all $t \in \mathbb{T}_{0}$. Similarly to (3.8), we have

$$
u(t) \leqslant w(t), \quad \forall t \in \mathbb{T}_{0} .
$$

Define a function $z(t)$ by

$$
z(t)=\int_{t_{0}}^{t}\left[g\left(\varphi_{1}(s)\right) \omega^{q}(s)+h\left(\varphi_{2}(s)\right) \omega^{r}(s)+k(t, s)+\int_{t_{0}}^{s}\left[f\left(\varphi_{3}(\tau)\right) \omega^{m}(\tau)+\xi(\tau)\right] \Delta \tau\right] \Delta s
$$

for all $t \in \mathbb{T}_{0}$. Clearly $z(t) \geqslant 0$ nondecreasing function with $z\left(t_{0}\right)=0$. We can write (3.10) as the following:

$$
\omega^{p}(t) \leqslant a(t)+b(t) z(t), \quad \forall t \in \mathbb{T}_{0} .
$$

From (3.13) and using Lemma 2.7, we get

$$
\begin{aligned}
& \omega^{\mathrm{q}}(\mathrm{t}) \leqslant(\mathrm{a}(\mathrm{t})+\mathrm{b}(\mathrm{t}) z(\mathrm{t}))^{\mathrm{q} / \mathrm{p}}(1)^{(\mathrm{p}-\mathrm{q}) / \mathrm{p}} \leqslant \frac{\mathrm{p}-\mathrm{q}}{\mathrm{p}}+\frac{\mathrm{q}}{\mathrm{p}} \mathrm{a}(\mathrm{t})+\frac{\mathrm{q}}{\mathrm{p}} \mathrm{b}(\mathrm{t}) z(\mathrm{t}), \quad \forall \mathrm{t} \in \mathbb{T}_{0}, \\
& \omega^{r}(t) \leqslant(a(t)+b(t) z(t))^{r / p}(1)^{p-r / p} \leqslant \frac{p-r}{p}+\frac{r}{p} a(t)+\frac{r}{p} b(t) z(t), \quad \forall t \in \mathbb{T}_{0}, \\
& \omega^{m}(t) \leqslant(a(t)+b(t) z(t))^{m / p}(1)^{p-m / p} \leqslant \frac{p-m}{p}+\frac{m}{p} a(t)+\frac{m}{p} b(t) z(t), \quad \forall t \in \mathbb{T}_{0} .
\end{aligned}
$$


By differentiating (3.12), and Lemma 2.3, we obtain

$$
\begin{aligned}
z^{\Delta}(t)= & {\left[g\left(\varphi_{1}(t)\right) \omega^{q}(t)+h\left(\varphi_{2}(t)\right) \omega^{r}(t)+\int_{t_{0}}^{t}\left[f\left(\varphi_{3}(\tau)\right) \omega^{m}(\tau)+\xi(\tau)\right] \Delta \tau\right.} \\
& \left.+k(\sigma(t), t)+\int_{t_{0}}^{t} k^{\Delta}(t, s) \Delta s\right]
\end{aligned}
$$

for all $t \in \mathbb{T}_{0}$. From (3.17) and using (3.14), (3.15), (3.16), and using Lemma 2.3, we get

$$
\begin{aligned}
z^{\Delta}(t) \leqslant & {\left[g\left(\varphi_{1}(t)\right)\left(\frac{p-q}{p}+\frac{q}{p} a(t)+\frac{q}{p} b(t) z(t)\right)\right.} \\
& +h\left(\varphi_{2}(t)\right)\left(\frac{p-r}{p}+\frac{r}{p} a(t)+\frac{r}{p} b(t) z(t)\right) \\
& \left.+\int_{t_{0}}^{t}\left[f\left(\varphi_{1}(\tau)\right)\left(\frac{p-m}{p}+\frac{m}{p} a(\tau)+\frac{m}{p} b(\tau) z(\tau)\right)+\xi(\tau)\right] \Delta \tau+k(\sigma(t), t)+\int_{t_{0}}^{t} k^{\Delta}(t, \tau) \Delta \tau\right] \\
\leqslant & {\left[b(t)\left(\frac{q}{p} g\left(\varphi_{1}(t)\right)+\frac{r}{p} h\left(\varphi_{2}(t)\right)\right)+\frac{m}{p} \int_{t_{0}}^{t} f\left(\varphi_{3}(\tau)\right) b(\tau) d \tau\right] z(t) } \\
& +\left[g\left(\varphi_{1}(t)\right)\left(\frac{p-q}{p}+\frac{q}{p} a(t)\right)+h\left(\varphi_{2}(t)\right)\left(\frac{p-r}{p}+\frac{r}{p} a(t)\right)\right. \\
& \left.+\int_{t_{0}}^{t} f\left(\varphi_{3}(\tau)\right)\left(\frac{p-m}{p}+\frac{m}{p} a(\tau)\right) \Delta \tau+\int_{t_{0}}^{t} \xi(\tau) \Delta \tau+k(\sigma(t), t)+\int_{t_{0}}^{t} k^{\Delta}(t, \tau) \Delta \tau\right] \\
= & \eta_{1}(t) z(t)+\zeta_{1}(t)
\end{aligned}
$$

for all $\mathrm{t} \in \mathbb{T}_{0}$, where $\eta_{1}$ and $\zeta_{1}$ are defined as in (3.3) and (3.4), respectively. Now by a suitable application of Lemma 2.4 with $z\left(t_{0}\right)=0$, the inequality (3.18) gives us the following estimation

$$
z(t) \leqslant \int_{t_{0}}^{t} \zeta_{1}(s) \zeta_{1}(s) e_{\eta_{1}}(t, \sigma(s)) \Delta s
$$

for all $t \in \mathbb{T}_{0}$, we get the required inequality (3.2) from (3.11), (3.13), and (3.19). This completes the proof.

Theorem 3.2. In Theorem 3.1, let $\hat{a} \in \mathrm{C}_{\mathrm{rd}}\left(\mathbb{T}_{0}, \mathbb{R}^{+}\right)$be a nondecreasing function. If

$$
\begin{aligned}
u^{p}(t) \leqslant & \hat{a}^{p}(t)+b(t) \int_{t_{0}}^{t}\left[g\left(\varphi_{1}(s)\right) u^{q}\left(\varphi_{1}(s)\right)+h\left(\varphi_{2}(s)\right) u^{r}\left(\varphi_{2}(s)\right)+k(t, s)\right. \\
& \left.+\int_{t_{0}}^{s}\left[f\left(\varphi_{3}(\tau)\right) u^{m}\left(\varphi_{3}(\tau)\right)+\xi(\tau)\right] \Delta \tau\right] \Delta s,
\end{aligned}
$$

with the initial condition

$$
\begin{cases}u(t)=\phi(t), & \text { if } \mathrm{t} \in\left[\alpha, \mathrm{t}_{0}\right] \cap \mathbb{T} \\ \phi\left(\varphi_{i}(\mathrm{t})\right) \leqslant \hat{\mathrm{a}}(\mathrm{t}), & \text { if } \varphi_{\mathrm{i}}(\mathrm{t}) \leqslant \mathrm{t}_{0}, \forall \mathrm{t} \in \mathbb{T}_{0}, i=1,2,3\end{cases}
$$

where $\varphi_{i}(t) \in\left(\mathbb{T}_{0}, \mathbb{T}\right), \varphi_{i}(t)<t,-\infty<\alpha=\inf \left\{\varphi_{i}(t), t \in \mathbb{T}_{0}\right\} \leqslant t_{0}, \phi \in C_{r d}\left(\left[\alpha, t_{0}\right] \cap \mathbb{T}, \mathbb{R}^{+}\right)$, then

$$
\mathrm{u}(\mathrm{t}) \leqslant \hat{\mathrm{a}}(\mathrm{t})\left\{1+\mathrm{b}(\mathrm{t}) \int_{\mathrm{t}_{0}}^{\mathrm{t}} \hat{\zeta_{1}}(\mathrm{~s}) \mathrm{e}_{\hat{\mathrm{r}}_{1}}(\mathrm{t}, \sigma(\mathrm{s})) \Delta \mathrm{s}\right\}^{1 / \mathrm{p}}
$$

for all $\mathrm{t} \in \mathbb{T}_{0}$, where

$$
\hat{\eta_{1}}(t)=b(t)\left(\frac{q}{p} g\left(\varphi_{1}(t)\right) \hat{a}^{q-p}(t)+\frac{r}{p} h\left(\varphi_{2}(t)\right) a^{r-p}(t)\right)+\frac{m}{p} \int_{t_{0}}^{t} f\left(\varphi_{3}(\tau)\right) \hat{a}^{m-p}(t) b(\tau) \Delta \tau,
$$


and

$$
\begin{aligned}
\hat{\zeta}_{1}(t)= & g\left(\varphi_{1}(t)\right) \hat{a}^{q-p}(t)+h\left(\varphi_{2}(t)\right) \hat{a}^{r-p}(t)+\int_{t_{0}}^{t} f\left(\varphi_{3}(\tau)\right) \hat{a}^{m-p}(\tau) \Delta \tau \\
& +\int_{t_{0}}^{t} \xi(\tau) \hat{a}^{-p}(\tau) \Delta \tau+k\left(\sigma(t) \hat{a}^{-p}(t), t\right)+\int_{t_{0}}^{t} k^{\Delta}(t, \tau) \hat{a}^{-p}(\tau) \Delta \tau
\end{aligned}
$$

for all $\mathrm{t} \in \mathbb{T}_{0}$.

Proof. Since $c(t)>0$ and nondecreasing, by the previous discussion in Theorem 3.1, we can write (3.20) in the following form

$$
\begin{aligned}
\left(\frac{\omega(t)}{\hat{a}(t)}\right)^{p} \leqslant & 1+b(t) \int_{t_{0}}^{t}\left[g\left(\varphi_{1}(s)\right) \hat{a}^{q-p}(s)\left(\frac{\omega(s)}{\hat{a}(s)}\right)^{q}+h\left(\varphi_{2}(s)\right) \hat{a}^{r-p}(s)\left(\frac{\omega(s)}{\hat{a}(s)}\right)^{r}\right. \\
& +\hat{a}^{-p}(t) k(t, s)+\int_{t_{0}}^{s}\left[f\left(\varphi_{3}(\tau)\right) \hat{a}^{m-p}(\tau)\left(\frac{\omega(\tau)}{\hat{a}(\tau)}\right)^{m}+\hat{a}^{-p}(t) \xi(s) \Delta \tau\right] \Delta s .
\end{aligned}
$$

By using the inequality proved in Theorem 3.1, we get the desired inequality in (3.21). This completes the proof.

As a special case of Theorem 3.1 when $\mathbb{T}=\mathbb{R}$, we have the relation (2.1) and then we get the following result.

Corollary 3.3. Let $u, a, g, h, b, \xi, f \in C\left(I, \mathbb{R}^{+}\right), k(t, s), k^{\Delta}(t, s) \in C\left(I \times I, \mathbb{R}^{+}\right)$, and $p \neq 0, p>q>0$, $p>r>0, p>m>0, p, q, r, m$ be constants. If

$$
\begin{aligned}
u^{p}(t) \leqslant & a(t)+b(t) \int_{t_{0}}^{t}\left[g\left(\varphi_{1}(s)\right) u^{q}\left(\varphi_{1}(s)\right)+h\left(\varphi_{2}(s)\right) u^{r}\left(\varphi_{2}(s)\right)+k(t, s)\right. \\
& \left.+\int_{0}^{s}\left[f\left(\varphi_{3}(\tau)\right) u^{m}\left(\varphi_{3}(\tau)\right)+\xi(\tau)\right] d \tau\right] d s
\end{aligned}
$$

with the initial condition

$$
\begin{cases}\mathrm{u}(\mathrm{t})=\phi(\mathrm{t}), & \text { if } \mathrm{t} \in\left[\alpha, \mathrm{t}_{0}\right] \cap \mathbb{T} \\ \phi\left(\varphi_{i}(\mathrm{t})\right) \leqslant(\mathrm{a}(\mathrm{t}))^{1 / p}, & \text { if } \varphi_{i}(\mathrm{t}) \leqslant \mathrm{t}_{0}, \forall \mathrm{t} \in \mathbb{T}_{0}, i=1,2,3\end{cases}
$$

where $\varphi_{i}(t) \in(I, I), \varphi_{i}(t)<t,-\infty<\alpha=\inf \left\{\varphi_{i}(t), t \in I\right\} \leqslant t_{0}, \phi \in C\left(I, \mathbb{R}^{+}\right)$, then

$$
u(t) \leqslant\left\{a(t)+b(t) \int_{t_{0}}^{t} \exp \left(\int_{t_{0}}^{s} \tilde{\zeta_{1}}(s) \tilde{\eta_{1}}(\lambda) d \lambda\right) d s\right\}^{1 / p}
$$

for all $\mathrm{t} \in \mathrm{I}$, where

$$
\tilde{\eta_{1}}(t)=b(t)\left(\frac{q}{p} g\left(\varphi_{1}(t)\right)+\frac{r}{p} h\left(\varphi_{2}(t)\right)\right)+\frac{m}{p} \int_{t_{0}}^{t} f\left(\varphi_{3}(\tau)\right) b(\tau) d \tau,
$$

and

$$
\begin{aligned}
\tilde{\zeta}_{1}(t)= & g\left(\varphi_{1}(t)\right)\left(\frac{p-q}{p}+\frac{q}{p} a(t)\right)+h\left(\varphi_{2}(t)\right)\left(\frac{p-r}{p}+\frac{r}{p} a(t)\right) \\
& +\int_{t_{0}}^{t} f\left(\varphi_{3}(\tau)\right)\left(\frac{p-m}{p}+\frac{m}{p} a(\tau)\right) d \tau+\int_{t_{0}}^{t} \xi(\tau) d \tau+k(t, t)+\int_{t_{0}}^{t} \frac{\partial}{\partial t} k(t, \tau) d \tau
\end{aligned}
$$

for all $\mathrm{t} \in \mathrm{I}$. 
Remark 3.4. It is interesting to note that as a special case, if we put $k(t, s)=0, f(t)=0, \xi(t)=0, r=1$, and $\varphi_{1}(t)=\varphi_{2}(t)=t$, then the inequality given in Corollary 3.3 reduces to the inequality given in [25, Theorem $\left.1\left(a_{1}\right)\right]$.

Remark 3.5. It is interesting to note that as a special case, if $\mathbb{T}=\mathbb{R}, k(t, s)=0, f(t)=0, \xi(t)=0, r=1$ and $\varphi_{1}(t)=\varphi_{2}(t)=t$, then the inequality given in Theorem 3.2 reduces to the inequality given in [25, Theorem $\left.1\left(a_{2}\right)\right]$.

Remark 3.6. It is interesting to note that as a special case, if we put $t_{0}=0, k(t, s)=\hat{h}(s), f(t)=0, \xi(t)=0$, and $\varphi_{1}(t)=t-\tau$, with $\tau>0, \varphi_{2}(t)=t$, then Corollary 3.3 reduces to [16, Theorem 1].

As a special case of Theorem 3.1, if $\mathbb{T}=\mathbb{Z}$ and using the relations (2.2) and (2.3), we obtain the following discrete result.

Corollary 3.7. Assume that $\mathrm{u}(\mathrm{n}), \mathrm{g}(\mathrm{n}), \mathrm{h}(\mathrm{n}), \mathrm{f}(\mathrm{n}), \mathrm{a}(\mathrm{n}), \mathrm{b}(\mathrm{n})$ are nonnegative sequences defined on $\mathbb{N}_{0}$, and $\mathrm{k}(\mathrm{n}, \mathrm{s}), \Delta_{1} \mathrm{k}(\mathrm{n}, \mathrm{s})$ are nonnegative sequences defined on $\mathrm{E}=\left\{(\mathrm{m}, \mathrm{n}) \in \mathbb{N}_{0}^{2}: 0 \leqslant \mathrm{n} \leqslant \mathrm{m}<\infty\right\}$. If $\mathrm{u}(\mathrm{n})$ satisfies the following delay discrete inequality

$$
\begin{aligned}
u^{p}(n) \leqslant & \left.a(n)+b(n) \sum_{s=0}^{n-1}\left[g\left(s-\lambda_{1}\right)\right) u^{q}\left(s-\lambda_{1}\right)\right)+h\left(s-\lambda_{2}\right) u^{r}\left(s-\lambda_{2}\right)+k(n, s) \\
& \left.+\sum_{\tau=0}^{s-1}\left[f\left(\tau-\lambda_{3}\right) u^{m}\left(\tau-\lambda_{3}\right)+\xi(\tau)\right]\right], \forall n \in \mathbb{N}_{0}
\end{aligned}
$$

with the initial condition

$$
\begin{cases}u(n)=\phi(n), & \text { if } n \in\left\{-\lambda_{i}, \ldots,-1,0\right\}, i=1,2,3 \\ \phi\left(n-\lambda_{i}\right) \leqslant(a(t))^{1 / p}, & \text { if } n \in \mathbb{N}_{0}, \quad n-\lambda_{i} \leqslant 0, i=1,2,3\end{cases}
$$

where $p, q, r, m$ and $\lambda_{i}$ are constants, $p \neq 0, p>q>0, p>r>0, p>m>0, \lambda_{i} \in \mathbb{N}_{0}, \varphi(n) \in \mathbb{R}_{+}$, $\mathrm{n} \in\left\{-\lambda_{i}, \ldots,-1,0\right\}$, then

$$
u(n) \leqslant\left\{a(n)+b(n) \sum_{s=0}^{n-1} \bar{\zeta}_{1}(s) \prod_{\tau=0}^{s-1}\left[1+\overline{\eta_{1}}(\tau)\right]\right\}^{1 / p}
$$

for all $\mathrm{n} \in \mathbb{N}_{0}$, where

$$
\overline{\eta_{1}}(n)=b(n)\left(\frac{q}{p} g\left(n-\lambda_{1}\right)+\frac{r}{p} h\left(n-\lambda_{2}\right)\right)+\frac{m}{p} \sum_{s=0}^{n-1} f\left(s-\lambda_{3}\right) b(n),
$$

and

$$
\begin{aligned}
\bar{\zeta}_{1}(n)= & g\left(n-\lambda_{1}\right)\left(\frac{p-q}{p}+\frac{q}{p} a(n)\right)+h\left(n-\lambda_{2}\right)\left(\frac{p-r}{p}+\frac{r}{p} a(n)\right) \\
& +\sum_{s=0}^{n-1} f\left(s-\lambda_{1}\right)\left(\frac{p-m}{p}+\frac{m}{p} a(s)\right)+k(n+1, n)+\sum_{s=0}^{n-1} \Delta k(n, s)+\sum_{s=0}^{n-1} \xi(s)
\end{aligned}
$$

for all $\mathrm{n} \in \mathbb{N}_{0}$.

Remark 3.8. It is interesting to note that as a special case, if we put $k(n, s)=0, f(n)=0, \xi(n)=0, r=1$ and $\lambda_{1}=\lambda_{2}=0$, then the inequality given in Corollary 3.7 reduces to the inequality given in [25, Theorem $\left.3\left(c_{1}\right)\right]$.

Remark 3.9. It is interesting to note that as a special case, if $\mathbb{T}=\mathbb{Z}$ and using the relations (2.2) and (2.3), if we put $k(n, s)=0, f(n)=0, \xi(n)=0, r=1$ and $\lambda_{1}=\lambda_{2}=0$, then the inequality given in Theorem 3.2 reduces to the inequality given in $\left[25\right.$, Theorem $\left.3\left(c_{2}\right)\right]$. 
Remark 3.10. It is interesting to note that as a special case, if we put $k(n, s)=\hat{h}(n), f(n)=0, \xi(n)=0$, and $\varphi_{1}(n)=n-\tau$, with $\tau>0, \varphi_{2}(n)=n$, then Corollary 3.7 reduces to [16, Theorem 3].

Theorem 3.11. Let $u, a, g, h, \xi, b, f \in C_{r d}\left(\mathbb{T}_{0}, \mathbb{R}^{+}\right)$, and $k(t, s), k^{\Delta}(t, s) \in C_{r d}\left(\mathbb{T}_{0} \times \mathbb{T}_{0}, \mathbb{R}^{+}\right)$, and $p \neq 0$, $\mathrm{p}>\mathrm{q}>0, \mathrm{p}>\mathrm{r}>0, \mathrm{p}>\mathrm{m}>0, \mathrm{p}, \mathrm{q}, \mathrm{r}, \mathrm{m}$ be constants. If

$$
\begin{aligned}
u^{p}(t) \leqslant & a(t)+b(t) \int_{t_{0}}^{t} k(t, s)\left[g\left(\varphi_{1}(s)\right) u^{q}\left(\varphi_{1}(s)\right)+h\left(\varphi_{2}(s)\right) u^{r}\left(\varphi_{2}(s)\right)\right. \\
& \left.+\int_{0}^{s}\left[f\left(\varphi_{3}(\tau)\right) u^{m}\left(\varphi_{3}(\tau)\right)+\xi(\tau)\right] \Delta \tau\right] \Delta s
\end{aligned}
$$

with the initial condition

$$
\begin{cases}u(t)=\phi(t), & \text { if } t \in\left[\alpha, t_{0}\right] \cap \mathbb{T} \\ \phi\left(\varphi_{i}(t)\right) \leqslant(a(t))^{1 / p}, & \text { if } \varphi_{i}(t) \leqslant t_{0}, \forall t \in \mathbb{T}_{0}, i=1,2,3\end{cases}
$$

where $\varphi_{\mathfrak{i}}(\mathrm{t}) \in\left(\mathbb{T}_{0}, \mathbb{T}\right), \varphi_{\mathfrak{i}}(\mathrm{t})<\mathrm{t},-\infty<\alpha=\inf \left\{\varphi_{\mathfrak{i}}(\mathrm{t}), \mathrm{t} \in \mathbb{T}_{0}\right\} \leqslant \mathrm{t}_{0}, \phi \in \mathrm{C}_{\mathrm{rd}}\left(\left[\alpha, \mathrm{t}_{0}\right] \cap \mathbb{T}, \mathbb{R}^{+}\right)$, then

$$
\omega(t) \leqslant\left\{a(t)+b(t) \int_{t_{0}}^{t} e_{\eta_{2}}(t, \sigma(s)) \zeta_{2}(s) \Delta s\right\}^{1 / p}
$$

for all $\mathrm{t} \in \mathbb{T}_{0}$, where

$$
\begin{aligned}
\eta_{2}(t)= & k(\sigma(t), t)\left[b(t)\left(\frac{q}{p} g\left(\varphi_{1}(t)\right)+\frac{r}{p} h\left(\varphi_{2}(t)\right)\right)+\frac{m}{p} \int_{t_{0}}^{t} f\left(\varphi_{3}(\tau)\right) b(\tau) \Delta \tau\right] \\
& +\int_{t_{0}}^{t} k^{\Delta}(t, s)\left(b(t)\left(\frac{q}{p} g\left(\varphi_{1}(s)\right)+\frac{r}{p} h\left(\varphi_{2}(s)\right)\right)+\frac{m}{p} \int_{t_{0}}^{s} f\left(\varphi_{3}(\tau)\right) b(\tau) \Delta \tau\right) \Delta s,
\end{aligned}
$$

and

$$
\begin{aligned}
\zeta_{2}(t)= & k(\sigma(t), t)\left[g\left(\varphi_{1}(t)\right)\left(\frac{p-q}{p}+\frac{q}{p} a(t)\right)+h\left(\varphi_{2}(t)\right)\left(\frac{p-r}{p}+\frac{r}{p} a(t)\right)\right. \\
& \left.+\int_{t_{0}}^{t} f\left(\varphi_{3}(\tau)\right)\left(\frac{p-m}{p}+\frac{m}{p} a(\tau)\right) \Delta \tau+\int_{t_{0}}^{t} \xi(\tau) \Delta \tau\right] \\
& +\int_{t_{0}}^{t} k^{\Delta}(t, s)\left[g\left(\varphi_{1}(s)\right)\left(\frac{p-q}{p}+\frac{q}{p} a(s)\right)+h\left(\varphi_{2}(s)\right)\left(\frac{p-r}{p}+\frac{r}{p} a(s)\right)\right. \\
& \left.+\int_{t_{0}}^{s} f\left(\varphi_{3}(\tau)\right)\left(\frac{p-m}{p}+\frac{m}{p} a(\tau)\right)+\int_{t_{0}}^{t} \xi(\tau) \Delta \tau\right] \Delta s .
\end{aligned}
$$

Proof. Fixing an arbitrary number $\mathrm{T}^{*} \in \mathbb{T}_{0}$, we define a function $\omega(\mathrm{t})$ by:

$$
\begin{aligned}
\omega(t)= & {\left[a\left(T^{*}\right)+b(t) \int_{t_{0}}^{t} k(t, s)\left[g\left(\varphi_{1}(s)\right) u^{\mathrm{q}}\left(\varphi_{1}(s)\right)+h\left(\varphi_{2}(s)\right) u^{r}\left(\varphi_{2}(s)\right)\right.\right.} \\
& \left.\left.+\int_{0}^{s}\left[f\left(\varphi_{3}(\tau)\right) u^{m}\left(\varphi_{3}(\tau)\right)+\xi(\tau)\right] \Delta \tau\right] \Delta s\right]^{1 / p} .
\end{aligned}
$$

Similar to the proof of Theorem 3.1, we easily obtain that $\omega(t)$ is a nonnegative and nondecreasing function, and we have

$$
\begin{aligned}
& u(t) \leqslant w(t), t \in \mathbb{T}_{0}, \\
& u\left(\varphi_{i}(t)\right) \leqslant \omega(t), \quad t \in \mathbb{T}_{0}, i=1,2,3,
\end{aligned}
$$


and

$$
\begin{aligned}
\omega^{p}(t) \leqslant & a(t)+b(t) \int_{t_{0}}^{t} k(t, s)\left[g\left(\varphi_{1}(s)\right) \omega^{q}(s)+h\left(\varphi_{2}(s)\right) \omega^{r}(s)\right. \\
& \left.+\int_{t_{0}}^{s}\left[f\left(\varphi_{3}(\tau)\right) \omega^{m}(\tau)+\xi(\tau)\right] \Delta \tau\right] \Delta s .
\end{aligned}
$$

Define a function $z(t)$ by

$$
z(t)=\int_{t_{0}}^{t} k(t, s)\left[g\left(\varphi_{1}(s)\right) \omega^{q}(s)+h\left(\varphi_{2}(s)\right) \omega^{r}(s)+\int_{t_{0}}^{s}\left[f\left(\varphi_{3}(\tau)\right) \omega^{m}(\tau)+\xi(\tau)\right] \Delta \tau\right] \Delta s,
$$

then $z(t) \geqslant 0$, and is nondecreasing with $z\left(t_{0}\right)=0$. As in the proof of Theorem 3.1, from (3.26), we see that the inequalities (3.14), (3.15), and (3.16) hold. By differentiating (3.27), and by using Lemma 2.3, we obtain

$$
\begin{aligned}
z^{\Delta}(\mathrm{t})= & \int_{\mathrm{t}_{0}}^{\mathrm{t}} k^{\Delta}(\mathrm{t}, \mathrm{s})\left[g\left(\varphi_{1}(\mathrm{~s})\right) \omega^{\mathrm{q}}(\mathrm{s})+\mathrm{h}\left(\varphi_{2}(\mathrm{~s})\right) \omega^{\mathrm{r}}(\mathrm{s})\right. \\
& \left.+\int_{\mathrm{t}_{0}}^{s}\left[\mathrm{f}\left(\varphi_{3}(\tau)\right) \omega^{\mathrm{m}}(\tau)+\xi(\tau)\right] \Delta \tau\right] \Delta s \\
& +k(\sigma(\mathrm{t}), \mathrm{t})\left[g\left(\varphi_{1}(\mathrm{t})\right) \omega^{\mathrm{q}}(\mathrm{t})+h\left(\varphi_{2}(\mathrm{t})\right) \omega^{\mathrm{r}}(\mathrm{t})+\int_{\mathrm{t}_{0}}^{\mathrm{t}}\left[\mathrm{f}\left(\varphi_{3}(\tau)\right) \omega^{\mathrm{m}}(\tau)+\xi(\tau)\right] \Delta \tau\right]
\end{aligned}
$$

for all $t \in \mathbb{T}_{0}$. From (3.28), and using (3.14), (3.15), and (3.16), we get

$$
\begin{aligned}
z^{\Delta}(t) \leqslant & {\left[k ( \sigma ( t ) , t ) \left[b(t)\left(\frac{q}{p} g\left(\varphi_{1}(t)\right)+\frac{r}{p} h\left(\varphi_{2}(t)\right)\right)\right.\right.} \\
& \left.+\frac{m}{p} \int_{t_{0}}^{t} f\left(\varphi_{3}(\tau)\right) b(\tau) \Delta \tau\right]+\int_{t_{0}}^{t} k^{\Delta}(t, s)\left(b(t)\left(\frac{q}{p} g\left(\varphi_{1}(s)\right)+\frac{r}{p} h\left(\varphi_{(} s\right)\right)\right) \\
& \left.\left.+\frac{m}{p} \int_{t_{0}}^{s} f\left(\varphi_{3}(\tau)\right) b(\tau) \Delta \tau\right) \Delta s\right] z(t) \\
& +k(\sigma(t), t)\left[g\left(\varphi_{1}(t)\right)\left(\frac{p-q}{p}+\frac{q}{p} a(t)\right)+h\left(\varphi_{2}(t)\right)\left(\frac{p-r}{p}+\frac{r}{p} a(t)\right)\right. \\
& \left.+\int_{t_{0}}^{t} f\left(\varphi_{3}(\tau)\right)\left(\frac{p-m}{p}+\frac{m}{p} a(\tau)\right) \Delta \tau+\int_{t_{0}}^{t} \xi(\tau) \Delta \tau\right] \\
& +\int_{t_{0}}^{t} k^{\Delta}(t, s)\left[g\left(\varphi_{1}(s)\right)\left(\frac{p-q}{p}+\frac{q}{p} a(s)\right)+h\left(\varphi_{2}(s)\right)\left(\frac{p-r}{p}+\frac{r}{p} a(s)\right)\right. \\
& \left.+\int_{t_{0}}^{s} f\left(\varphi_{3}(\tau)\right)\left(\frac{p-m}{p}+\frac{m}{p} a(\tau)\right) \Delta \tau+\int_{t_{0}}^{t} \xi(\tau) \Delta \tau\right] \Delta s \\
= & \eta_{2}(t) z(t)+\zeta_{2}(t), \forall t \in \mathbb{T}_{0},
\end{aligned}
$$

where $\eta_{2}(t)$ and $\zeta_{2}(t)$ are defined in (3.23) and (3.24), respectively. From the inequality (3.29) and by using Lemma 2.4 with $z\left(t_{0}\right)=0$, the inequality (3.29) gives us the following estimation

$$
z(t) \leqslant \int_{t_{0}}^{t} e_{\eta_{2}}(t, \sigma(s)) \zeta_{2}(s) \Delta s, \forall t \in \mathbb{T}_{0} .
$$

By using (3.30) in $\omega^{p}(t) \leqslant a(t)+b(t) z(t)$, we get the required inequality in (3.22). This completes the proof. 
Theorem 3.12. In Theorem 3.11, let $\hat{\mathrm{a}} \in \mathrm{C}_{\mathrm{rd}}\left(\mathbb{T}_{0}, \mathbb{R}^{+}\right)$be a nondecreasing function. If

$$
\begin{aligned}
u^{p}(t) \leqslant & \hat{a}^{p}(t)+b(t) \int_{t_{0}}^{t} k(t, s)\left[g\left(\varphi_{1}(s)\right) u^{q}\left(\varphi_{1}(s)\right)+h\left(\varphi_{2}(s)\right) u^{r}\left(\varphi_{2}(s)\right)\right. \\
& \left.+\int_{t_{0}}^{s}\left[f\left(\varphi_{3}(\tau)\right) u^{m}\left(\varphi_{3}(\tau)\right)+\xi(\tau)\right] \Delta \tau\right] \Delta s
\end{aligned}
$$

for all $\mathrm{t} \in \mathbb{T}_{0}$, with the initial condition

$$
\begin{cases}\mathrm{u}(\mathrm{t})=\phi(\mathrm{t}), & \text { if } \mathrm{t} \in\left[\alpha, \mathrm{t}_{0}\right] \cap \mathbb{T}, \\ \phi\left(\varphi_{\mathrm{i}}(\mathrm{t})\right) \leqslant \hat{\mathrm{a}}(\mathrm{t}), & \text { if } \varphi_{\mathrm{i}}(\mathrm{t}) \leqslant \mathrm{t}_{0}, \forall \mathrm{t} \in \mathbb{T}_{0}, \mathfrak{i}=1,2,3,\end{cases}
$$

where $\varphi_{i}(t) \in\left(\mathbb{T}_{0}, \mathbb{T}\right), \varphi_{i}(t)<t,-\infty<\alpha=\inf \left\{\varphi_{i}(t), t \in \mathbb{T}_{0}\right\} \leqslant t_{0}, \phi \in C_{r d}\left(\left[\alpha, t_{0}\right] \cap \mathbb{T}, \mathbb{R}^{+}\right)$, then

$$
u(t) \leqslant \hat{a}(t)\left\{1+b(t) \int_{t_{0}}^{t} e_{\hat{\eta}_{2}}(t, \sigma(s)) \hat{\zeta}_{2}(s) \Delta s\right\}^{1 / p}
$$

for all $\mathrm{t} \in \mathbb{T}_{0}$, where

$$
\begin{aligned}
\hat{\eta}_{2}(t)= & k(\sigma(t), t)\left[b(t)\left(\frac{q}{p} g\left(\varphi_{1}(t)\right) \hat{a}^{q-p}(t)+\frac{r}{p} h\left(\varphi_{2}(t)\right) \hat{a}^{r-p}(t)\right)\right. \\
& \left.+\frac{m}{p} \int_{t_{0}}^{t} f\left(\varphi_{3}(\tau)\right) \hat{a}^{m-p}(\tau) b(\tau) \Delta \tau\right] \\
& +\int_{t_{0}}^{t} k^{\Delta}(t, s)\left(b(t)\left(\frac{q}{p} g\left(\varphi_{1}(s)\right) \hat{a}^{q-p}(s)+\frac{r}{p} h\left(\varphi_{2}(s)\right) \hat{a}^{r-p}(s)\right)\right. \\
& \left.+\frac{m}{p} \int_{t_{0}}^{s} f\left(\varphi_{3}(\tau)\right) \hat{a}^{m-p}(\tau) b(\tau) \Delta \tau\right) \Delta s,
\end{aligned}
$$

and

$$
\begin{aligned}
\hat{\zeta}_{2}(t)= & k(\sigma(t), t)\left[g\left(\varphi_{1}(t)\right) \hat{a}^{q-p}(t)+h\left(\varphi_{2}(t)\right) \hat{a}^{r-p}(t)\right. \\
& \left.+\int_{t_{0}}^{t} f\left(\varphi_{3}(\tau)\right) \hat{a}^{m-p}(\tau) d \tau+\int_{t_{0}}^{t} \hat{a}^{-p} \xi(\tau) \Delta \tau\right] \\
& +\int_{t_{0}}^{t} k^{\Delta}(t, s)\left[g\left(\varphi_{1}(s)\right) \hat{a}^{q-p}(s)+h\left(\varphi_{2}(s)\right) \hat{a}^{r-p}(s)\right. \\
& \left.+\int_{t_{0}}^{s} f\left(\varphi_{3}(\tau)\right) \hat{a}^{m-p}(\tau) \Delta \tau\right] \Delta s+\int_{t_{0}}^{t} \hat{a}^{-p} \xi(\tau) \Delta \tau
\end{aligned}
$$

for all $\mathrm{t} \in \mathbb{T}_{0}$.

Proof. Since $\hat{a}(t)>0$ and nondecreasing, as the previous discussion in Theorem (3.11), we can write (3.31) as in the following form

$$
\begin{aligned}
\left(\frac{\omega(t)}{\hat{a}(t)}\right)^{p} \leqslant & 1+b(t) \int_{t_{0}}^{t} k(t, s)\left[g\left(\varphi_{1}(s)\right) \hat{a}^{q-p}(s)\left(\frac{\omega(s)}{a(s)}\right)^{q}+h\left(\varphi_{2}(s)\right) \hat{a}^{r-p}(s)\left(\frac{\omega(s)}{\hat{a}(s)}\right)^{r}\right. \\
& \left.+\int_{0}^{s}\left[f\left(\varphi_{3}(\tau)\right) \hat{a}^{m-p}(\tau)\left(\frac{\omega(\tau)}{\hat{a}(\tau)}\right)^{m}+\hat{a}^{-p} \xi(\tau)\right] \Delta \tau\right] d s
\end{aligned}
$$

for all $t \in \mathbb{T}_{0}$. Applying the inequality given in Theorem 3.11 yields the desired result in (3.32). This completes the proof. 
As a special case of Theorem 3.11 when $\mathbb{T}=\mathbb{R}$ we have the relation (2.1) and then we get the following result.

Corollary 3.13. Let $u, a, g, h, \xi, b, f \in C\left(I, \mathbb{R}^{+}\right), k(t, s), k^{\Delta}(t, s) \in C\left(I \times I, \mathbb{R}^{+}\right)$, and $p \neq 0, p>q>0$, $p>r>0, p>m>0, p, q, r, m$ be constants. If

$$
\begin{aligned}
u^{p}(t) \leqslant & a(t)+b(t) \int_{t_{0}}^{t} k(t, s)\left[g\left(\varphi_{1}(s)\right) u^{q}\left(\varphi_{1}(s)\right)+h\left(\varphi_{2}(s)\right) u^{r}\left(\varphi_{2}(s)\right)\right. \\
& \left.+\int_{0}^{s}\left[f\left(\varphi_{3}(\tau)\right) u^{m}\left(\varphi_{3}(\tau)\right)+\xi(\tau)\right] d \tau\right] d s
\end{aligned}
$$

with the initial condition

$$
\begin{cases}u(t)=\phi(t), & \text { if } t \in\left[\alpha, t_{0}\right] \cap \mathbb{T}, \\ \phi\left(\varphi_{i}(t)\right) \leqslant(a(t))^{1 / p}, & \text { if } \varphi_{i}(t) \leqslant t_{0}, \forall t \in \mathbb{T}_{0}, i=1,2,3\end{cases}
$$

where $\varphi_{i}(t) \in(I, I), \varphi_{i}(t)<t,-\infty<\alpha=\inf \left\{\varphi_{i}(t), t \in I \leqslant t_{0}, \phi \in C\left(I, \mathbb{R}^{+}\right)\right.$, then

$$
\omega(t) \leqslant a(t)+b(t) \int_{t_{0}}^{t} \tilde{\zeta_{2}}(s) \exp \left(\int_{t_{0}}^{s} \tilde{\eta_{2}}(\tau) d \tau\right) d s
$$

for all $\mathrm{t} \in \mathrm{I}$, where

$$
\begin{aligned}
\tilde{\eta_{2}}(t)= & k(t, t)\left[b(t)\left(\frac{q}{p} g\left(\varphi_{1}(t)\right)+\frac{r}{p} h\left(\varphi_{2}(t)\right)\right)+\frac{m}{p} \int_{t_{0}}^{t} f\left(\varphi_{3}(\tau)\right) b(\tau) d \tau\right] \\
& +\int_{t_{0}}^{t} \frac{\partial}{\partial t} k(t, s)\left(b(t)\left(\frac{q}{p} g\left(\varphi_{1}(s)\right)+\frac{r}{p} h\left(\varphi_{2}(s)\right)\right)+\frac{m}{p} \int_{t_{0}}^{s} f\left(\varphi_{3}(\tau)\right) b(\tau) d \tau\right) d s,
\end{aligned}
$$

and

$$
\begin{aligned}
\tilde{\tau_{2}}(t)= & k(t, t)\left[g\left(\varphi_{1}(t)\right)\left(\frac{p-q}{p}+\frac{q}{p} a(t)\right)+h\left(\varphi_{2}(t)\right)\left(\frac{p-r}{p}+\frac{r}{p} a(t)\right)\right. \\
& \left.+\int_{t_{0}}^{t}\left(f\left(\varphi_{3}(\tau)\right)\left(\frac{p-m}{p}+\frac{m}{p} a(\tau)\right) d \tau+\xi(\tau)\right) d \tau\right] \\
& +\int_{t_{0}}^{t} k^{\Delta}(t, s)\left[g\left(\varphi_{1}(s)\right)\left(\frac{p-q}{p}+\frac{q}{p} a(s)\right)+h\left(\varphi_{2}(s)\right)\left(\frac{p-r}{p}+\frac{r}{p} a(s)\right)\right. \\
& \left.+\int_{t_{0}}^{s}\left(f\left(\varphi_{3}(\tau)\right)\left(\frac{p-m}{p}+\frac{m}{p} a(\tau)\right)+\xi(\tau)\right) d \tau\right] d s .
\end{aligned}
$$

Remark 3.14. It is interesting to note that as a special case, if we put $f(t)=0, \xi(t)=0, r=1, b(t)=b(t)$, and $\varphi_{1}(t)=\varphi_{2}(t)=t$, then the inequality given in Corollary 3.13 reduces to the inequality given in $[25$, Theorem $\left.1\left(a_{3}\right)\right]$.

As a special case of Theorem 3.11, if $\mathbb{T}=\mathbb{Z}$ and using the relations (2.2) and (2.3), we obtain the following discrete result.

Corollary 3.15. Assume that $\mathrm{u}(\mathrm{n}), \mathrm{g}(\mathrm{n}), \mathrm{h}(\mathrm{n}), \mathrm{b}(\mathrm{n}), \mathrm{f}(\mathrm{n}), \mathrm{a}(\mathrm{n})$ are nonnegative sequences defined on $\mathbb{N}_{0}$, and $k(n, s), \Delta_{1} k(n, s)$ are nonnegative sequences defined on $E=\left\{(m, n) \in \mathbb{N}_{0}^{2}: 0 \leqslant n \leqslant m<\infty 1\right\}$. If $u(n)$ satisfies the following delay discrete inequality

$$
\left.u^{p}(n) \leqslant a(n)+b(n) \sum_{s=0}^{n-1} k(n, s)\left[g\left(s-\lambda_{1}\right)\right) u^{q}\left(s-\lambda_{1}\right)\right)+h\left(s-\lambda_{2}\right) u^{r}\left(s-\lambda_{2}\right)
$$




$$
+\sum_{\tau=0}^{s-1}\left[f\left(\tau-\lambda_{3}\right) u^{m}\left(\tau-\lambda_{3}\right)+\xi(\tau)\right], \forall n \in \mathbb{N}_{0},
$$

with the initial condition

$$
\begin{cases}u(n)=\phi(n), & \text { if } n \in\left\{-\lambda_{i}, \ldots,-1,0\right\}, i=1,2,3, \\ \phi\left(n-\lambda_{i}\right) \leqslant(a(t))^{1 / p}, & \text { if } n \in \mathbb{N}_{0}, \quad n-\lambda_{i} \leqslant 0, i=1,2,3,\end{cases}
$$

where $p, q, r, m$ and $\lambda_{i}$ are constants, $p \neq 0, p>q>0, p>r>0, p>m>0, \lambda_{i} \in \mathbb{N}_{0}, \varphi(n) \in \mathbb{R}_{+}$, $n \in\left\{-\lambda_{i}, \ldots,-1,0\right\}$, then

$$
u(n) \leqslant\left\{a(n)+b(n) \sum_{s=0}^{n-1} \overline{\zeta_{2}}(s) \prod_{\tau=0}^{s-1}\left[1+\overline{\eta_{2}}(\tau)\right]\right\}^{1 / p}
$$

for all $\mathrm{n} \in \mathbb{N}_{0}$, where

$$
\begin{aligned}
\overline{\eta_{2}}(t)= & k(n+1, n)\left[b(n)\left(\frac{q}{p} g\left(n-\lambda_{1}\right)+\frac{r}{p} h\left(n-\lambda_{2}\right)\right)+\frac{m}{p} \sum_{s=0}^{n-1} f\left(s-\lambda_{3}\right) b(n)\right] \\
& +\sum_{s=1}^{n} \Delta k(n, s)\left(b(n)\left(\frac{q}{p} g\left(s-\lambda_{1}\right)+\frac{r}{p} h\left(s-\lambda_{2}\right)\right)+\frac{m}{p} \sum_{\tau=0}^{s-1} f\left(\tau-\lambda_{3}\right) b(\tau)\right),
\end{aligned}
$$

and

$$
\begin{aligned}
\bar{\zeta}_{2}(t)= & k(n+1, n)\left[g\left(n-\lambda_{1}\right)\left(\frac{p-q}{p}+\frac{q}{p} a(n)\right)+h\left(n-\lambda_{2}\right)\left(\frac{p-r}{p}+\frac{r}{p} a(n)\right)\right. \\
& \left.+\sum_{s=0}^{n-1}\left(f\left(s-\lambda_{3}\right)\left(\frac{p-m}{p}+\frac{m}{p} a(s)\right)+\xi(s)\right)\right] \\
& +\sum_{s=0}^{n-1} \Delta k(n, s)\left[g\left(s-\lambda_{1}\right)\left(\frac{p-q}{p}+\frac{q}{p} a(s)\right)+h\left(s-\lambda_{2}\right)\left(\frac{p-r}{p}+\frac{r}{p} a(s)\right)\right. \\
& \left.+\sum_{\tau=0}^{s-1}\left(f\left(\tau-\lambda_{3}\right)\left(\frac{p-m}{p}+\frac{m}{p} a(\tau)\right)+\xi(\tau) d \tau\right)\right] .
\end{aligned}
$$

Remark 3.16. It is interesting to note that as a special case, if we put $f(n)=0, \xi(n)=0, r=1$, and $\lambda_{1}=\lambda_{2}=0$, then the inequality given in Corollary 3.15 reduces to the inequality given in [25, Theorem 3 $\left.\left(c_{3}\right)\right]$.

Theorem 3.17. Let $u, a, g, \xi, b, f \in C_{r d}\left(\mathbb{T}_{0}, \mathbb{R}^{+}\right)$, and $k(t, s), k^{\Delta}(t, s) \in C_{r d}\left(\mathbb{T}_{0}, \mathbb{R}^{+}\right) p \neq 0, p>q>0$, $\mathrm{p}>\mathrm{r}>0, \mathrm{p}>\mathrm{m}>0, \mathrm{p}, \mathrm{q}, \mathrm{r}, \mathrm{m}$ be constants, and $\mathrm{L} \in \mathrm{C}_{\mathrm{rd}}\left(\mathbb{T}_{0} \times \mathbb{R}^{+}, \mathbb{R}^{+}\right)$such that

$$
0 \leqslant \mathrm{~L}(\mathrm{t}, \mathrm{x})-\mathrm{L}(\mathrm{t}, \mathrm{y}) \leqslant \mathrm{M}(\mathrm{t}, \mathrm{y})(\mathrm{x}-\mathrm{y}), \forall \mathrm{t} \in \mathbb{T}_{0},
$$

where $\mathrm{n} \in \mathrm{C}_{\mathrm{rd}}\left(\mathbb{T}_{0} \times \mathbb{R}^{+}, \mathbb{R}^{+}\right)$, and $\mathrm{x} \geqslant 0, \mathrm{y} \geqslant 0$. If

$$
\begin{aligned}
u(t) \leqslant & a(t)+b(t) \int_{t_{0}}^{t}\left[g\left(\varphi_{1}(s)\right) u^{q}\left(\varphi_{1}(s)\right)+L\left(s, u^{r}\left(\varphi_{2}(s)\right)\right)+k(t, s)\right. \\
& \left.+\int_{t_{0}}^{s}\left[f\left(\varphi_{3}(\tau)\right) u^{m}\left(\varphi_{3}(\tau)\right)+\xi(\tau)\right] \Delta \tau\right] \Delta s
\end{aligned}
$$

with the initial condition

$$
\begin{cases}\mathrm{u}(\mathrm{t})=\phi(\mathrm{t}), & \text { if } \mathrm{t} \in\left[\alpha, \mathrm{t}_{0}\right] \cap \mathbb{T}, \\ \phi\left(\varphi_{\mathrm{i}}(\mathrm{t})\right) \leqslant \mathrm{a}(\mathrm{t}), & \text { if } \varphi_{\mathrm{i}}(\mathrm{t}) \leqslant \mathrm{t}_{0}, \forall \mathrm{t} \in \mathbb{T}_{0}, \mathrm{i}=1,2,3,\end{cases}
$$


where $\varphi_{\mathfrak{i}}(\mathrm{t}) \in\left(\mathbb{T}_{0}, \mathbb{T}\right), \varphi_{\mathfrak{i}}(\mathrm{t})<\mathrm{t},-\infty<\alpha=\inf \left\{\varphi_{\mathfrak{i}}(\mathrm{t}), \mathrm{t} \in \mathbb{T}_{0}\right\} \leqslant \mathrm{t}_{0}, \phi \in \mathrm{C}_{\mathrm{rd}}\left(\left[\alpha, \mathrm{t}_{0}\right] \cap \mathbb{T}, \mathbb{R}^{+}\right)$, then

$$
u(t) \leqslant a(t)+b(t) \int_{t_{0}}^{t} \zeta_{4}(s) e_{\eta_{4}}(t, \sigma(s)) \zeta_{4}(s) \Delta s
$$

for all $\mathrm{t} \in \mathbb{T}_{0}$, where

$$
\eta_{4}(t)=\frac{q}{p} g\left(\varphi_{1}(t)\right) b(t)+\frac{r}{p} n\left(t, \frac{p-r}{p}+\frac{r}{p} a(t)\right) b(t)+\frac{m}{p} \int_{t_{0}}^{t} f\left(\varphi_{3}(\tau)\right) b(t, \tau) \Delta \tau,
$$

and

$$
\begin{aligned}
\zeta_{4}(t)= & g\left(\varphi_{1}(t)\right)\left(\frac{p-q}{p}+\frac{q}{p} a(t)\right)+L\left(t, \frac{p-r}{p}+\frac{r}{p} a(t)\right)+\int_{t_{0}}^{t} f\left(\varphi_{3}(\tau)\right)\left(\frac{p-m}{p}+\frac{m}{p} a(\tau)\right) \Delta \tau \\
& +\int_{t_{0}}^{t} \xi(\tau) \Delta \tau+k(\sigma(t), t)+\int_{t_{0}}^{t} k^{\Delta}(t, \tau) \Delta \tau
\end{aligned}
$$

for all $\mathrm{t} \in \mathbb{T}_{0}$.

Proof. Fixing an arbitrary number $\mathrm{T}^{*} \in \mathbb{T}_{0}$, we define a function $\omega(\mathrm{t})$ by:

$$
\begin{aligned}
w(t)= & {\left[a\left(T^{*}\right)+b(t) \int_{t_{0}}^{t}\left[g\left(\varphi_{1}(s)\right) u^{q}\left(\varphi_{1}(s)\right)+L\left(s, u^{r}\left(\varphi_{2}(s)\right)\right)+k(t, s)\right.\right.} \\
& \left.\left.+\int_{t_{0}}^{s}\left[f\left(\varphi_{3}(\tau)\right) u^{m}\left(\varphi_{3}(\tau)\right)+\xi(\tau)\right] \Delta \tau\right] \Delta s\right]^{1 / p}
\end{aligned}
$$

Similar to the proof of Theorem 3.1 with the initial condition (3.35), we easily obtain that $\omega(t)$ is a nonnegative and nondecreasing function, and we have

$$
\begin{aligned}
u(t) \leqslant w(t), & t \in \mathbb{T}_{0}, \\
u\left(\varphi_{i}(t)\right) \leqslant \omega(t), & t \in \mathbb{T}_{0}, i=1,2,3,
\end{aligned}
$$

and

$$
\omega(t) \leqslant a(t)+b(t) \int_{t_{0}}^{t}\left[g\left(\varphi_{1}(s)\right) \omega^{q}(s)+h\left(s, \omega^{r}(s)\right)+k(t, s)+\int_{t_{0}}^{s}\left[f\left(\varphi_{3}(\tau)\right) \omega^{m}(\tau)+\xi(\tau)\right] \Delta \tau\right] \Delta s .
$$

Define a function $z(t)$ by

$$
\begin{aligned}
z(t)= & \int_{t_{0}}^{t}\left[g\left(\varphi_{1}(s)\right) \omega^{q}(s)+L\left(s, \omega^{r}(s)\right)+k(t, s)\right. \\
& \left.+\int_{t_{0}}^{s}\left[f\left(\varphi_{3}(\tau)\right) \omega^{m}(\tau)+\xi(\tau)\right] \Delta \tau\right] \Delta s .
\end{aligned}
$$

Then $z(t)>0$, and is a nondecreasing function with $z\left(t_{0}\right)=0$. As in the proof of Theorem 3.1 from (3.34), we see that the inequalities (3.14), (3.15), and (3.16) hold. By differentiating (3.39), and using Lemma 2.3, we obtain

$$
z^{\Delta}(\mathrm{t})=\left[g\left(\varphi_{1}(\mathrm{t})\right) \omega^{\mathrm{q}}(\mathrm{t})+\mathrm{L}\left(\mathrm{t}, \omega^{\mathrm{r}}(\mathrm{t})\right)+\int_{\mathrm{t}_{0}}^{\mathrm{t}}\left[\mathrm{f}\left(\varphi_{3}(\tau)\right) \omega^{\mathrm{m}}(\tau)+\xi(\tau)\right] \Delta \tau+\mathrm{k}(\sigma(\mathrm{t}), \mathrm{t})+\int_{\mathrm{t}_{0}}^{\mathrm{t}} k^{\Delta}(\mathrm{t}, \tau) \Delta \tau\right]
$$


for all $t \in \mathbb{T}_{0}$. From (3.40) and using (3.14), (3.15), (3.16), and (3.33), we have

$$
\begin{aligned}
z^{\Delta}(t) \leqslant & {\left[g\left(\varphi_{1}(t)\right)\left(\frac{p-q}{p}+\frac{q}{p} a(t)+\frac{q}{p} b(t) z(t)\right)+L\left(t, \frac{p-r}{p}+\frac{r}{p} a(t)+\frac{r}{p} b(t) z(t)\right)\right.} \\
& -L\left(t, \frac{p-r}{p}+\frac{r}{p} a(t)\right)+L\left(t, \frac{p-r}{p}+\frac{r}{p} a(t)\right) \\
& +\int_{t_{0}}^{t} f\left(\varphi_{3}(\tau)\right)\left(\frac{p-m}{p}+\frac{m}{p} a(\tau)+\frac{m}{p} b(\tau) z(\tau)\right) \Delta \tau \\
& \left.+\int_{t_{0}}^{t} \xi(\tau) \Delta \tau+k(\sigma(t), t)+\int_{t_{0}}^{t} k^{\Delta}(t, \tau) \Delta \tau\right] \\
\leqslant & \frac{q}{p} g(t) b(t)+\frac{r}{p} n\left(t, \frac{p-r}{p}+\frac{r}{p} a(t)\right) b(t) \\
& \left.+\frac{m}{p} \int_{t_{0}}^{t} f(\tau) b(\tau) \Delta \tau\right] z(t)+\left[g\left(\varphi_{1}(t)\right)\left(\frac{p-q}{p}+\frac{q}{p} a(t)\right)\right. \\
& +L\left(t, \frac{p-r}{p}+\frac{r}{p} a(t)\right)+\int_{t_{0}}^{t} f\left(\varphi_{3}(\tau)\right)\left(\frac{p-m}{p}+\frac{m}{p} a(\tau)\right) \Delta \tau \\
& \left.+\int_{t_{0}}^{t} \xi(\tau) \Delta \tau+k(\sigma(t), t)+\int_{t_{0}}^{t} k^{\Delta}(t, \tau) \Delta \tau\right] \\
= & \eta_{4}(t) z(t)+\zeta_{4}(t), \quad \forall t \in \mathbb{T}_{0},
\end{aligned}
$$

where $\eta_{4}(t)$ and $\zeta_{4}(t)$ are defined in (3.37) and (3.38), respectively. From the inequality (3.41) and by using Lemma 2.4 with $z\left(t_{0}\right)=0$ we get,

$$
z(\mathrm{t}) \leqslant \int_{\mathrm{t}_{0}}^{\mathrm{t}} \zeta_{4}(\mathrm{~s}) e_{\mathrm{\eta} 4}(\mathrm{t}, \sigma(\mathrm{s})) \zeta_{4}(\mathrm{~s}) \Delta \mathrm{s}
$$

for all $t \in \mathbb{T}_{0}$. By using (3.42) in $\omega^{p}(t) \leqslant a(t)+b(t) z(t)$, the required inequality in (3.36) follows. This completes the proof.

As a special case of Theorem 3.1, when $\mathbb{T}=\mathbb{R}$ we have the relation (2.1) and we get the following result.

Corollary 3.18. Let $u, a, g, h, b, \xi, f \in C\left(I, \mathbb{R}^{+}\right)$, and $k(t, s), k^{\Delta}(t, s) \in C\left(I \times I, \mathbb{R}^{+}\right), p \neq 0, p>q>0$, $p>r>0, p>m>0, p, q, r, m$ be constants, and $L \in C\left(I \times \mathbb{R}^{+}, \mathbb{R}^{+}\right)$such that

$$
0 \leqslant L(t, x)-L(t, y) \leqslant M(t, y)(x-y), \forall t \in I,
$$

where $\mathrm{M} \in \mathrm{C}\left(\mathrm{I} \times \mathbb{R}^{+}, \mathbb{R}^{+}\right)$, and $\mathrm{x} \geqslant 0, \mathrm{y} \geqslant 0$. If

$$
\begin{aligned}
u(t) \leqslant & a(t)+b(t) \int_{t_{0}}^{t}\left[g\left(\varphi_{1}(s)\right) u^{q}\left(\varphi_{1}(s)\right)+L\left(t, u^{r}\left(\varphi_{2}(s)\right)\right)+k(t, s)\right. \\
& \left.+\int_{t_{0}}^{s}\left[f\left(\varphi_{3}(\tau)\right) u^{m}\left(\varphi_{3}(\tau)\right)+\xi(\tau)\right] d \tau\right] d s,
\end{aligned}
$$

with the initial condition

$$
\begin{cases}\mathrm{u}(\mathrm{t})=\phi(\mathrm{t}), & \text { if } \mathrm{t} \in\left[\alpha, \mathrm{t}_{0}\right] \cap \mathbb{T}, \\ \phi\left(\varphi_{\mathrm{i}}(\mathrm{t})\right) \leqslant \mathrm{a}(\mathrm{t}), & \text { if } \varphi_{\mathrm{i}}(\mathrm{t}) \leqslant \mathrm{t}_{0}, \forall \mathrm{t} \in \mathbb{T}_{0}, \mathrm{i}=1,2,3,\end{cases}
$$

where $\varphi_{i}(t) \in(I, I), \varphi_{i}(t)<t,-\infty<\alpha=\inf \left\{\varphi_{i}(t), t \in I \leqslant t_{0}, \phi \in C\left(I, \mathbb{R}^{+}\right)\right.$, then

$$
u(t) \leqslant\left\{a(t)+b(t) \int_{t_{0}}^{t} \tilde{\eta_{4}} \exp \left(\int_{t_{0}}^{s}(\lambda) d \lambda\right) \tilde{\zeta}_{4}(s) d s\right\}^{1 / p}
$$


for all $\mathrm{t} \in \mathrm{I}$, where

$$
\tilde{\eta_{4}}(t)=\frac{q}{p} g\left(\varphi_{1}(t)\right) b(t)+\frac{r}{p} M\left(t, \frac{p-r}{p}+\frac{r}{p} a(t)\right) b(t)+\frac{m}{p} \int_{t_{0}}^{t} f\left(\varphi_{3}(\tau)\right) b(\tau) d \tau,
$$

and

$$
\begin{aligned}
\tilde{\zeta}_{4}(t)= & g\left(\varphi_{1}(t)\right)\left(\frac{p-q}{p}+\frac{q}{p} a(t)\right)+L\left(t, \frac{p-r}{p}+\frac{r}{p} a(t)\right)+\int_{t_{0}}^{t} f\left(\varphi_{3}(\tau)\right)\left(\frac{p-m}{p}+\frac{m}{p} a(\tau)\right) d \tau \\
& +\int_{t_{0}}^{t} \xi(\tau) \Delta \tau+k(\sigma(t), t)+\int_{t_{0}}^{t} \frac{\partial}{\partial t} k(t, \tau) d \tau
\end{aligned}
$$

for all $\mathrm{t} \in \mathrm{I}$.

Remark 3.19. It is interesting to note that as a special case, if we put $k(t, s)=0, f(t)=0, \xi(t)=0, g(t)=0$, $\varphi_{2}(t)=t$, and $r=1$ then Corollary 3.18 reduces to [25, Theorem $\left.2\left(b_{1}\right)\right]$.

Remark 3.20. It is interesting to note that as a special case, if we put $t_{0}=0, k(t, s)=0, f(t)=0, \xi(t)=0$, $g(t)=0, \varphi_{2}(t)=t-s$ with $\tau>0$, and $r=1$ then Corollary 3.18 reduces to [16, Theorem 2].

As a special case of Theorem 3.1, if $\mathbb{T}=\mathbb{Z}$ and using the relations (2.2) and (2.3), we obtain the following discrete result.

Corollary 3.21. Assume that $\mathrm{u}(\mathrm{n}), \mathrm{g}(\mathrm{n}), \mathrm{h}(\mathrm{n}), \mathrm{f}(\mathrm{n}), \mathrm{b}(\mathrm{t}), \mathrm{a}(\mathrm{n})$ are nonnegative sequences defined on $\mathbb{N}_{0}$ and $k(n, s), \Delta_{1} k(n, s)$ are nonnegative sequences defined on $E=\left\{(m, n) \in \mathbb{N}_{0}^{2}: 0 \leqslant n \leqslant m<\infty\right.$. Suppose $\mathrm{L}: \mathbb{N}_{0} \times \mathbb{R}_{+} \rightarrow \mathbb{R}_{+}$be a function such that

$$
0 \leqslant L(n, x)-L(n, y) \leqslant M(n, y)(x-y)
$$

for $\mathrm{n} \in \mathbb{N}_{0}, x \geqslant y \geqslant 0$, where $M(n, y)$ is a real-valued nonnegative function defined for $n \in \mathbb{N}_{0}, y \in \mathbb{R}_{+}$. If $u(n)$ satisfies the following delay discrete inequality

$$
\begin{aligned}
u^{p}(n) \leqslant & \left.a(n)+b(n) \sum_{s=0}^{n-1}\left[g\left(s-\lambda_{1}\right)\right) u^{q}\left(s-\lambda_{1}\right)\right)+L\left(s, u^{r}\left(s-\lambda_{2}\right)\right)+k(n, s) \\
& \left.+\sum_{\tau=0}^{s-1}\left[f\left(\tau-\lambda_{3}\right) u^{m}\left(\tau-\lambda_{3}\right)+\xi(\tau)\right]\right]
\end{aligned}
$$

for $\mathrm{n} \in \mathbb{N}_{0}$, with the initial condition

$$
\begin{cases}u(n)=\phi(n), & \text { if } n \in\left\{-\lambda_{i}, \ldots,-1,0\right\}, i=1,2,3, \\ \phi\left(n-\lambda_{i}\right) \leqslant a(t), & \text { if } n \in \mathbb{N}_{0}, \quad n-\lambda_{i} \leqslant 0, i=1,2,3,\end{cases}
$$

where $p, q, r, m$ and $\lambda_{i}$ are constants, $p \neq 0, p>q>0, p>r>0, p>m>0, \lambda_{i} \in \mathbb{N}_{0}, \varphi(n) \in \mathbb{R}_{+}$, $\mathrm{n} \in\left\{-\lambda_{i}, \ldots,-1,0\right\}$, then

$$
u(n) \leqslant\left\{a(n)+b(n) \sum_{s=0}^{n-1} \bar{\zeta}_{4}(s) \prod_{\tau=0}^{s-1}\left[1+\overline{\eta_{4}}(\tau)\right]\right\}^{1 / p}
$$

for all $\mathrm{n} \in \mathbb{N}_{0}$, where

$$
\overline{\eta_{4}}(t)=\frac{q}{p} g\left(s-\lambda_{1}\right) b(t)+\frac{r}{p} M\left(t, \frac{p-r}{p}+\frac{r}{p} a(t)\right) b(t)+\frac{m}{p} \sum_{s=0}^{n-1} f\left(s-\lambda_{3}\right) b(\tau),
$$


and

$$
\begin{aligned}
\bar{\zeta}_{4}(t)= & g\left(n-\lambda_{1}\right)\left(\frac{p-q}{p}+\frac{q}{p} a(n)\right)+L\left(n, \frac{p-r}{p}+\frac{r}{p} a(n)\right)+\sum_{s=0}^{n-1} f\left(s-\lambda_{3}\right)\left(\frac{p-m}{p}+\frac{m}{p} a(s)\right) \\
& +\sum_{s=0}^{n-1} \xi(s)+k(n+1, n)+\sum_{s=0}^{n-1} \Delta_{1} k(n, s)
\end{aligned}
$$

for all $\mathrm{n} \in \mathbb{N}_{0}$.

Remark 3.22. It is interesting to note that as a special case, if we put $k(n, s)=0, f(n)=0, \xi(n)=0$, $g(n)=0, \lambda_{2}=n$, and $r=1$ then Corollary 3.21 reduces to [25, Theorem $\left.4\left(d_{1}\right)\right]$.

Remark 3.23. It is interesting to note that as a special case, if we put $t_{0}=0$ and put $k(n, s)=0, f(n)=0$, $\xi(n)=0, b(n)=b(n), g(n)=0, \varphi_{2}(n)=n-\tau$, with $\tau>0$ and $r=1$ then Corollary 3.21 reduces to [16, Theorem 4].

\section{Some applications}

In this section, we will present some applications for the results which we have established above and apply them to qualitative and quantitative analysis of solutions of certain delay dynamic equations on time scales to which the inequalities available in the literature do not apply directly.

Example 4.1. Consider the following delay dynamic integral equation on time scales:

$$
\left(u^{p}\right)^{\Delta}(t)=\Lambda\left(t, u(t), u\left(\varphi(t), \int_{t_{0}}^{t} \Xi(\varphi(s), u(\varphi(s)) \Delta s)\right), \quad t \in \mathbb{T}_{0}\right.
$$

with the initial condition

$$
\begin{cases}u(t)=\phi(t), & t \in\left[\alpha, t_{0}\right] \cap \mathbb{T} \\ \phi(\varphi(t)) \leqslant|C|^{1 / p}, & \varphi(t) \leqslant t_{0}, \forall t \in \mathbb{T}_{0}\end{cases}
$$

where $\varphi(t) \in\left(\mathbb{T}_{0}, \mathbb{T}\right), \varphi(t)<t,-\infty<\alpha=\inf \left\{\varphi(t), t \in \mathbb{T}_{0}\right\} \leqslant t_{0}, \phi \in C_{\text {rd }}\left(\left[\alpha, t_{0}\right] \cap \mathbb{T}, \mathbb{R}^{+}\right)$, and $\left.u \in C\left(\mathbb{T}_{0}, \mathbb{R}^{+}\right), \Lambda \in C_{\text {rd }}\left(\mathbb{T}_{0} \times \mathbb{R}^{3}, \mathbb{R}\right), \Xi \in[\alpha, \infty] \cap \mathbb{T} \times \mathbb{R}, \mathbb{R}\right)$ and $C=u^{p}\left(t_{0}\right)$.

Theorem 4.2. Assume that

$$
\begin{aligned}
\left|\Lambda\left(t, u(t), u\left(\varphi(t), \int_{t_{0}}^{t} \Xi(\varphi(s), u(\varphi(s))) \Delta s\right)\right)\right| \leqslant & g(\varphi(t)) u^{p}(\varphi(t))+h(\varphi(t)) u^{p}(\varphi(t)) \\
& +k(t, s)+\Xi(\varphi(t), u(\varphi(t)))
\end{aligned}
$$

and

$$
\Xi(\varphi(t), u(\varphi(t))) \leqslant f(\varphi(t)) u^{p}(\varphi(t))+\xi(t),
$$

where $u, a, g, h, \xi, f \in C_{r d}\left(\mathbb{T}_{0}, \mathbb{R}^{+}\right), k(t, s), k^{\Delta}(t, s) \in C_{r d}\left(\mathbb{T}_{0} \times \mathbb{T}_{0}, \mathbb{R}^{+}\right)$, and $p \neq 0, p>q>0, p>r>0$, $p>m>0, p, q, r, m$ are constants. If $u$ is a solution of the retarded dynamic equation (4.1) satisfying the initial condition (4.2), then

$$
u(t) \leqslant\left\{C+\int_{t_{0}}^{t} \delta(s) e_{\gamma}(t, \sigma(s)) \Delta s\right\}^{1 / p}
$$

for all $\mathrm{t} \in \mathbb{T}_{0}$, where

$$
\gamma(\mathrm{t})=\left(\frac{\mathrm{q}}{\mathrm{p}} \mathrm{g}(\varphi(\mathrm{t}))+\frac{\mathrm{r}}{\mathrm{p}} \mathrm{h}(\varphi(\mathrm{t}))\right)+\frac{\mathrm{m}}{\mathrm{p}} \int_{\mathrm{t}_{0}}^{\mathrm{t}} \mathrm{f}(\varphi(\tau)) \Delta \tau
$$


and

$$
\begin{aligned}
\delta(t)= & g(\varphi(t))\left(\frac{p-q}{p}+\frac{q C}{p}\right)+h(\varphi(t))\left(\frac{p-r}{p}+\frac{r C}{p}\right)+\int_{t_{0}}^{t} f(\varphi(\tau))\left(\frac{p-m}{p}+\frac{m C}{p}\right) \Delta \tau \\
& +\int_{t_{0}}^{t} \xi(\tau) \Delta \tau+k(\sigma(t), t)+\int_{t_{0}}^{t} k^{\Delta}(t, \tau) \Delta \tau
\end{aligned}
$$

for all $\mathrm{t} \in \mathbb{T}_{0}$.

Proof. Clearly, the solution $u$ of Equation (2.7) with the initial Condition (4.2) satisfies the equivalent delay dynamic equation on time scales

$$
\mathrm{u}^{\mathrm{p}}(\mathrm{t})=\mathrm{C}+\int_{\mathrm{t}_{0}}^{\mathrm{t}} \wedge\left(\mathrm{s}, \mathrm{u}(\mathrm{s}), \mathrm{u}\left(\varphi(\mathrm{s}), \int_{\mathrm{t}_{0}}^{\mathrm{s}} \Xi(\varphi(\tau), \mathrm{u}(\varphi(\tau))) \Delta \tau\right)\right) \Delta \mathrm{s}
$$

for all $t \in \mathbb{T}_{0}$ with the initial Condition (4.2). In fact, from (4.6) and by using the assumptions (4.3) and (4.4), we have

$$
\begin{aligned}
\left|u^{p}(t)\right|= & \left.\mid C+\int_{t_{0}}^{t} \Lambda\left(s, u(s), u\left(\varphi(s), \int_{t_{0}}^{s} \Xi(\varphi(\tau)), u(\varphi(\tau))\right) \Delta \tau\right)\right) \Delta s \mid \\
\leqslant & \left.|C|+\int_{t_{0}}^{t} \mid \Lambda\left(s, u(s), u\left(\varphi(s), \int_{t_{0}}^{s} \Xi(\varphi(\tau)), u(\varphi(\tau))\right) \Delta \tau\right)\right) \Delta s \mid \\
\leqslant & |C|+\int_{t_{0}}^{t}\left[g(\varphi(s))\left|u^{q}(\varphi(s))\right|+h(\varphi(s))\left|u^{r}(\varphi(s))\right|+k(t, s)\right. \\
& \left.+\int_{t_{0}}^{s}\left[f(\varphi(\tau))\left|u^{m}(\varphi(\tau))\right|+\xi(\tau)\right] \Delta \tau\right] \Delta s,
\end{aligned}
$$

with the initial condition (4.2). Then a suitable application of Theorem 3.1 (with $\varphi_{1}=\varphi_{2}=\varphi_{3}=\varphi$, $a^{p}(t)=C$ and $\left.b(t)=1\right)$ to (4.7), yields the desired Estimate (4.5) for solutions of Equation (4.1). We note that, the right hand side of (4.5) gives us the bound on the solution of (4.1) in terms of the known quantities. This completes the proof.

\section{Acknowledgment}

The last author's research was supported by a HKU Outstanding Teaching Award.

The authors would like to thank the referees for their extremely careful reading and thoughtful suggestions.

\section{References}

[1] A. Abdeldaim, A. A. El-Deeb, On generalized of certain retarded nonlinear integral inequalities and its applications in retarded integro-differential equations, Appl. Math. Comput., 256 (2015), 375-380. 1

[2] A. Abdeldaim, A. A. El-Deeb, On some new nonlinear retarded integral inequalities with iterated integrals and their applications in integro-differential equations, British J. Math. Comput. Science, 5 (2015), 479-491.

[3] A. Abdeldaim, A. A. El-Deeb, On some generalizations of certain retarded nonlinear integral inequalities with iterated integrals and an application in retarded differential equation, J. Egyptian Math. Soc., 23 (2015), 470-475.

[4] A. Abdeldaim, A. A. El-Deeb, Some new retarded nonlinear integral inequalities with iterated integrals and their applications in retarded differential equations and integral equations, J. Fract. Calc. Appl., 5 (2014), 9 pages. 1

[5] E. Akin-Bohner, M. Bohner, F. Akin, Pachpatte inequalities on time scales, JIPAM J. Inequal. Pure Appl. Math., 6 (2005), 1443-5756. 1

[6] D. R. Anderson, Dynamic double integral inequalities in two independent variables on time scales, J. Math. Inequal., 2 (2008), 163-184. 1

[7] R. Bellman, The stability of solutions of linear differential equations, Duke Math. J., 10 (1943), 643-647. 1 
[8] I. Bihari, A generalization of a lemma of Bellman and its application to uniqueness problems of differential equations, Acta Math. Acad. Sci. Hungar., 7 (1956), 81-94. 1

[9] M. Bohner, A. Peterson, Advances in dynamic equations on time scales, Birkhäuser Boston, Boston, (2003). 1

[10] M. Bohner, A. Peterson, Dynamic equations on time scales, Birkhäuser Boston, Boston, (2001). 1, 1, 2, 2.1, 2.2, 2.3, $2.4,2.5,2.6$

[11] H. M. El-Owaidy, A. A. Ragab, A. A. El-Deeb, W. M. K. Abuelela, On some new nonlinear integral inequalities of Gronwall-Bellman type, Kyungpook Math. J., 54 (2014), 555-575. 1

[12] T. H. Gronwall, Note on the derivatives with respect to a parameter of the solutions of a system of differential equations, Ann. of Math., 20 (1919), 292-296. 1

[13] S. Hilger, Analysis on measure chains-a unified approach to continuous and discrete calculus, Results Math., 18 (1990), 18-56. 1

[14] W. N. Li, Some new dynamic inequalities on time scales, J. Math. Anal. Appl., 319 (2006), 802-814. 1

[15] W. N. Li, Some Pachpatte type inequalities on time scales, Comput. Math. Appl., 57 (2009), 275-282. 1

[16] W. N. Li, Some nonlinear delay integral inequalities and their analogues, An. Şt. Univ. Ovidius Constanţa Ser. Mat., 18 (2010), 149-160. 1, 1, 1, 3.6, 3.10, 3.20, 3.23

[17] W. N. Li, M. Han, Bounds for certain nonlinear dynamic inequalities on time scales, Discrete Dyn. Nat. Soc., 2009 (2009), 14 pages. 1

[18] W. N. Li, Q. X. Zhang, F. Qiu, Some nonlinear delay discrete inequalities and their applications, Demonstratio Math., 39 (2006), 771-782.

[19] A. Liu, M. Bohner, Gronwall-OuIang-type integral inequalities on time scales, J. Inequal. Appl., 2010 (2010), 15 pages. 1

[20] Q.-H. Ma, J. Pečarić, Explicit bounds on some new nonlinear retarded integral inequalities and their applications, Taiwanese J. Math., 13 (2009), 287-306. 1

[21] D. S. Mitrinovic, P. M. Vasic, Analytic inequalities, Springer, Berlin, (1970). 2.7

[22] B. G. Pachpatte, Explicit bounds on certain integral inequalities, J. Math. Anal. Appl., 267 (2002), 48-61. 1

[23] B. G. Pachpatte, Inequalities for differential and integral equations, Academic Press, San Diego, (1998).

[24] B. G. Pachpatte, Integral and finite difference inequalities and applications, Elsevier, (2006). 1

[25] B. G. Pachpatte, On some new inequalities related to a certain inequality arising in the theory of differential equations, J. Math. Anal. Appl., 251 (2000), 736-751. 1, 1, 1, 1, 3.4, 3.5, 3.8, 3.9, 3.14, 3.16, 3.19, 3.22

[26] B. G. Pachpatte, On some fundamental integral inequalities and their discrete analogues, JIPAM. J. Inequal. Pure Appl. Math., 2 (2001), 13 pages. 1

[27] S. H. Saker, Some nonlinear dynamic inequalities on time scales and applications, J. Math. Inequal., 4 (2010), 561-579. 1

[28] F.-H. Wong, C.-C. Yeh, C.-H. Hong, Gronwall inequalities on time scales, Math. Inequal. Appl., 9 (2006), $75-86$.

[29] L. Yin, Q.-M. Luo, F. Qi, Several integral inequalities on time scales, J. Math. Inequal., 6 (2012), 419-429. 1 